ARTICLE

https://doi.org/10.1038/s41467-019-10716-w

\title{
A striatal interneuron circuit for continuous target pursuit
}

\author{
Namsoo Kim¹, Haofang E. Li ${ }^{1}$, Ryan N. Hughes ${ }^{1}$, Glenn D.R. Watson ${ }^{1}$, David Gallegos ${ }^{2}$, Anne E. West (10 ${ }^{2}$, \\ II Hwan $\mathrm{Kim}^{3}$ \& Henry H. Yin ${ }^{1,2}$
}

Most adaptive behaviors require precise tracking of targets in space. In pursuit behavior with a moving target, mice use distance to target to guide their own movement continuously. Here, we show that in the sensorimotor striatum, parvalbumin-positive fast-spiking interneurons (FSIs) can represent the distance between self and target during pursuit behavior, while striatal projection neurons (SPNs), which receive FSI projections, can represent selfvelocity. FSIs are shown to regulate velocity-related SPN activity during pursuit, so that movement velocity is continuously modulated by distance to target. Moreover, bidirectional manipulation of FSI activity can selectively disrupt performance by increasing or decreasing the self-target distance. Our results reveal a key role of the FSI-SPN interneuron circuit in pursuit behavior and elucidate how this circuit implements distance to velocity transformation required for the critical underlying computation.

\footnotetext{
${ }^{1}$ Department of Psychology and Neuroscience, Duke University, Durham, NC 27708, USA. ${ }^{2}$ Department of Neurobiology, Duke University, Durham, NC 27708, USA. ${ }^{3}$ Department of Anatomy and Neurobiology, University of Tennessee Health and Science Center, Memphis, TN 27708, USA. Correspondence and requests for materials should be addressed to H.H.Y. (email: hy43@duke.edu)
} 
W hether pursuing a prey or approaching a mate, natural behaviors often involve continuous tracking of targets in space. Yet the neural substrates of such pursuit behavior remain poorly understood. Technical limitations have prevented the study of natural pursuit behavior in freely moving animals ${ }^{1-4}$. In this study, we designed a new behavioral task, in which mice follow a continuously moving target that delivers sucrose reward. Using 3D motion capture, we were able to track not only the position of the mouse but also the distance to target, a crucial variable for accurate pursuit. This task allows us to compare continuous behavioral variables and neural activity recorded at the same time.

We studied the contribution of the inhibitory interneuron circuit in the sensorimotor striatum to pursuit behavior. The striatum is a major basal ganglia (BG) nucleus that has been implicated in motor control, compulsive behavior, and habit formation $^{5-9}$. A critical circuit in the striatum is formed by the parvalbumin-positive GABAergic fast-spiking interneurons (FSIs) and striatal projection neurons (SPNs) ${ }^{10,11}$. FSIs, which constitute $<1 \%$ of the striatal neuronal population, receive glutamatergic inputs from the cerebral cortex and project to many SPNs, which make up over $90 \%$ of the population ${ }^{12,13}$. Reduced number of FSIs is associated with neuropsychiatric disorders, such as Tourette's syndrome and obsessive-compulsive disorder ${ }^{14,15}$. Behavioral studies have also implicated the FSIs in choice behavior and habitual lever pressing ${ }^{13,16}$.

Recent work has shown that sensorimotor SPN activity is often highly correlated with movement velocity ${ }^{17,18}$, and optogenetic activation of SPNs alters movement velocity in a frequencydependent fashion ${ }^{19}$. Yet it remains unclear how, during target pursuit behavior, the striatal output is commanded by representation of distance to target. We hypothesize that the FSI-SPN interneuron circuit plays a critical role in pursuit behavior, allowing cortical inputs representing the spatial relationship between self and target to reach the BG. In particular, the distance representation can be converted into instantaneous velocity commands during pursuit behavior. To test this hypothesis, we used wireless in vivo electrophysiology, calcium imaging, and celltype-specific manipulation of neural activity to examine the contributions of the striatal microcircuit to pursuit behavior in freely moving mice. Our results established for the first time a key role of this local striatal circuit in pursuit behavior.

\section{Results}

Behavioral task. We recorded head and target positions during pursuit behavior using motion capture at 100 frames per second (Fig. 1a, b). All mice rapidly learned to follow the moving target (Supplementary Fig. 1, Supplementary movie 1) and showed comparable performance whether the target velocity is constant $(16 \mathrm{~mm} / \mathrm{s}$, leftward or rightward) or variable $(5-48 \mathrm{~mm} / \mathrm{s})$. Because pursuit behavior is self-initiated in our task, we separated periods in which the mice followed the target and periods in which they did not. 'Following' is defined as staying in a spatially defined zone close to the target for at least $800 \mathrm{~ms}(<15 \mathrm{~mm}$ $x$-axis, $<10 \mathrm{~mm}$ in $y$-axis, $<20 \mathrm{~mm}$ in $z$-axis), when the head is next to the sucrose spout. Otherwise, behavior is classified as 'not following' (Fig. 1b-d; for variable velocity see Supplementary Fig. 1). When the animal is not following, it could be engaged in any number of activities, such as grooming, orienting, rearing, and so on. A cross-correlation analysis suggests that mice use distance information to adjust their velocity (Fig. 1e). With distance as the reference variable, a positive lag means that distance leads velocity, and that the distance representation is used to guide velocity. This is exactly what we found. This lag measure is also positively correlated with the distance, the key measure of pursuit performance: the worse the pursuit performance, the more self-velocity lags self-target distance, as expected if distance is used to adjust velocity.

Neuronal representations of distance and velocity. To elucidate how striatal neurons contribute to pursuit behavior, we recorded striatal neurons while mice performed the task (453 neurons from 24 mice). We classified FSIs and SPNs based on their different average firing rates and spike waveforms. We identified 362 putative SPNs in the dorsolateral striatum (trough to peak width: $570.8 \pm 2.3 \mu$ s, firing rate: $2.1 \pm 0.1 \mathrm{~Hz}$ ) and 91 FSIs (width: 173.9 $\pm 4.9 \mu$ s, firing rate: $20.4 \pm 0.9 \mathrm{~Hz}$, Supplementary Fig. 2). The proportion of FSIs is higher than expected from anatomical studies $(1-2 \%)$ because these neurons have much higher firing rates compared to SPNs.

FSIs and SPNs showed distinct representations of behavioral variables: FSIs more commonly represented self-target distance (56\% of recorded) whereas SPNs (32\%) more commonly represented velocity (chi square $=88.71, P<0.0001$, Supplementary Tables 1-4, Supplementary Fig. 3). As shown in Fig. 2, there are two distinct populations of SPNs: One population increases firing as leftward velocity increases (Fig. 2a-c) and the other increases firing as rightward velocity increases (Fig. 2d-f). When we analyzed the relationship between single unit activity and velocity from the entire session, the correlation coefficient. By comparison, correlation with other variables is much lower (Fig. 2g, Supplementary Fig. 4). Using the population average activity as a measure of the ensemble activity, there is strong correlation between neural activity and velocity (Fig. $2 \mathrm{~h}$ ). Overall, the relationship between neural activity and velocity can be described with a sigmoid function ${ }^{20}$.

Our results suggest that the ensemble activity of many neurons with similar velocity representations (e.g. leftward) is used as the signal for velocity command. With negative feedback control, the actual velocity achieved closely matches the command. Interestingly, the correlation with velocity is significantly reduced when the mice are not following, for both populations of velocitycorrelated neurons (Fig. 2i). This finding suggests that the relationship between velocity and SPN output is contingent upon self-initiated pursuit behavior.

On the other hand, many FSIs showed continuous representation of target distance (Fig. 3, Supplementary Fig. 5). Their firing rates varied monotonically with target distance. The representation of target distance is independent of target or self-movement direction (whether leftward or rightward). One group increases firing as left distance increases, and decreases firing as right distance increases (Fig. 3a-c), whereas a second group shows the opposite pattern (Fig. 3d-f). By comparison, the correlation with other variables is much lower (Fig. 3g). There is a strong linear relationship between the population activity of distance FSIs and distance (Fig. 3h). For both populations, the mean firing rate corresponds to roughly the 'straight ahead' condition. For the 'left+' neurons, the highest firing rate is reached at the leftmost extreme and lowest firing rate at the rightmost extreme, and vice versa for the 'right+' neurons. Figure $3 i$ shows that the correlation with distance is significantly reduced when the mice are not following.

Because the striatum contains many different types of neurons, classification of a relatively rare class like the FSI based purely on firing rate and spike waveform may not be reliable. There are other interneurons that also have higher firing rates than SPNs ${ }^{12}$. To confirm our classification, we used optrodes to selectively stimulate and record PV+ FSIs at the same time. We injected a Cre-dependent channelrhodopsin (DIO-ChR2) in transgenic mice that express Cre-recombinase 
a

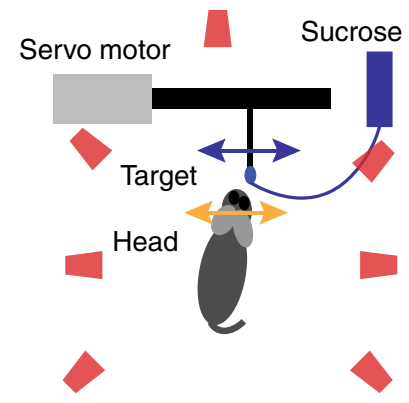

Osprey camera

Target position

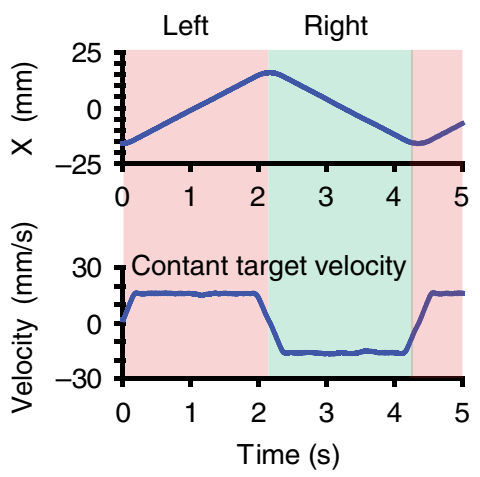

e

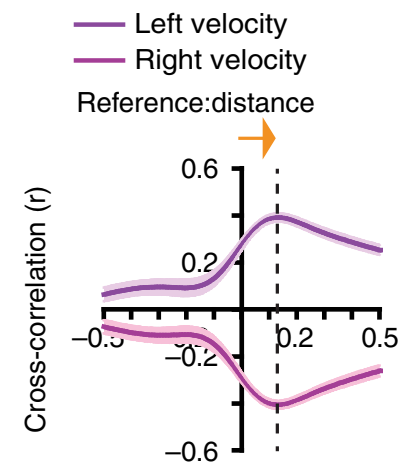

Time (s) b
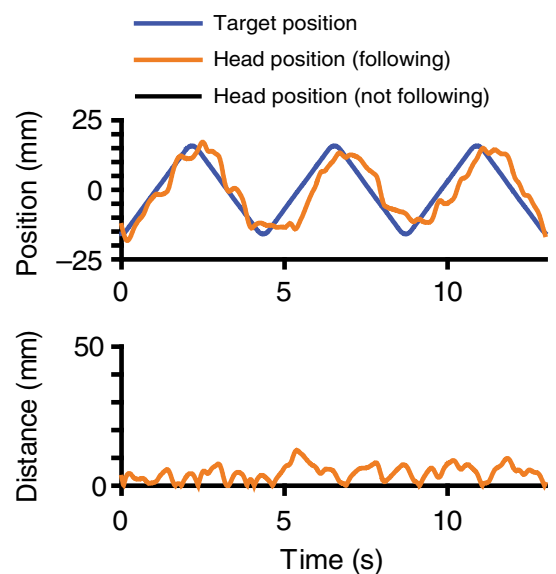

C
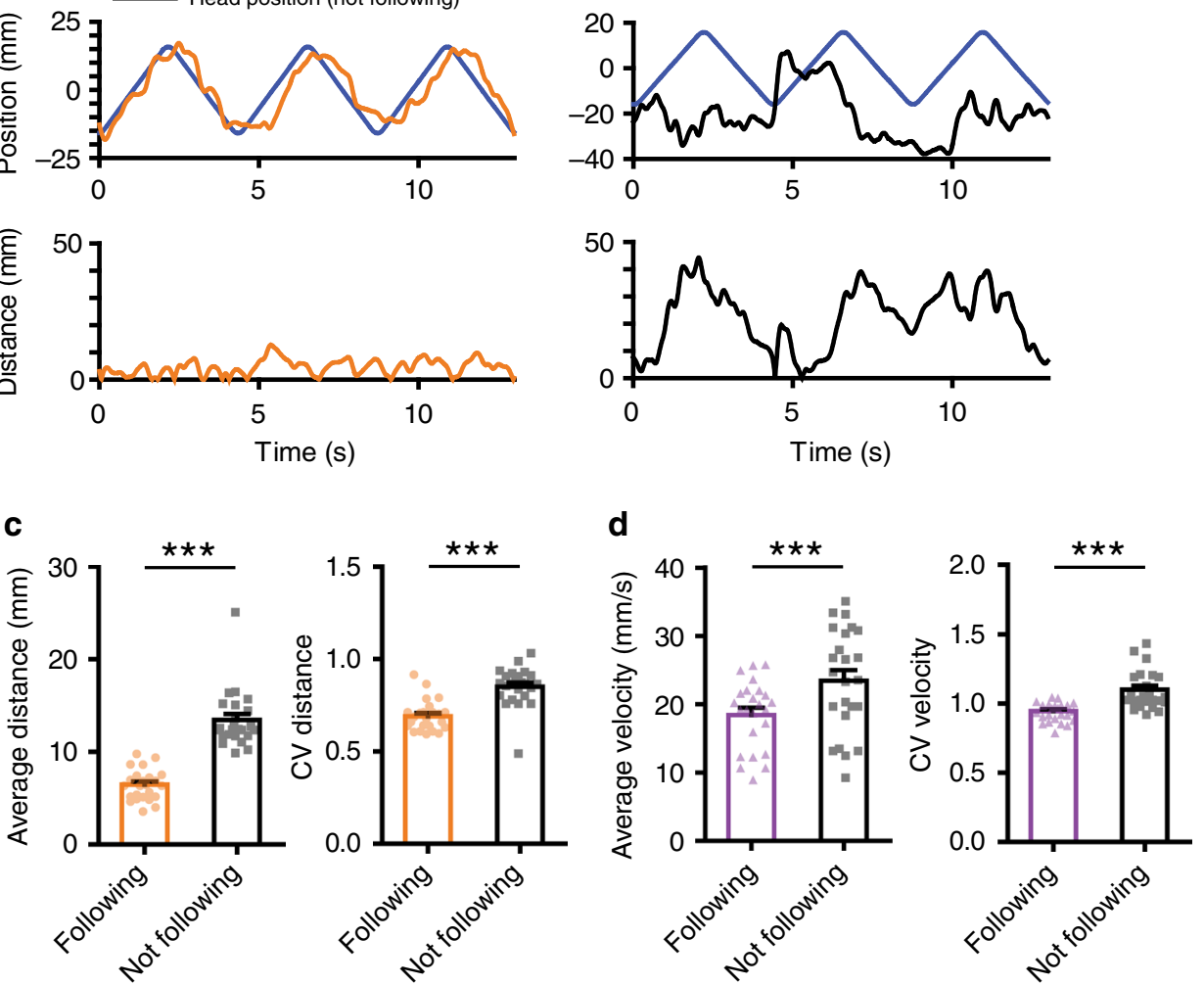

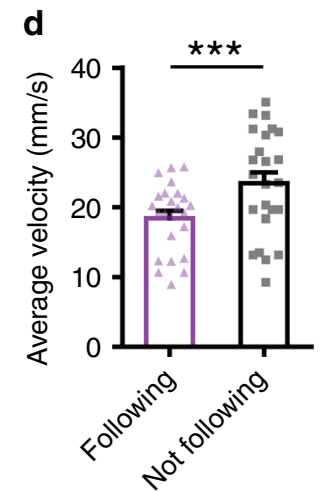

Not following

Time (s)

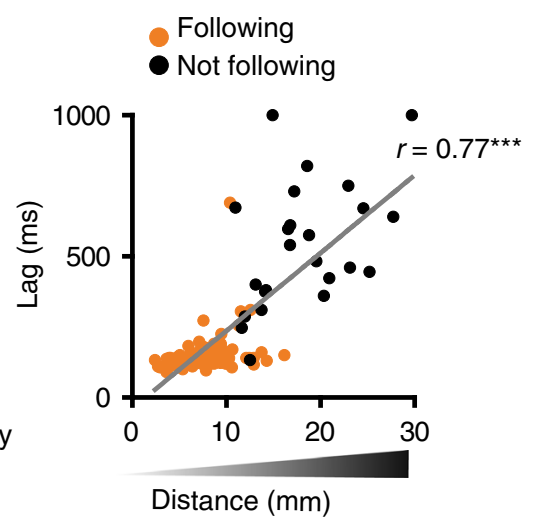

Fig. 1 Summary of behavioral results. a Top, illustration of the pursuit task and location of the infrared cameras for 3D motion capture. The target moves continuously from side to side, each cycle consisting of both leftward and rightward movements. During pursuit, the target could be on either side of the mouse. Bottom, position and velocity traces of the target, which moves along the $x$-axis at a constant velocity of $16 \mathrm{~mm} / \mathrm{s}$ (see extended Fig. 1 for variable velocity condition). The total distance traveled from left to right is $32 \mathrm{~mm}$. Thus, it takes the target $\sim 2 \mathrm{~s}$ to move from one extreme to another.

b Representative traces of mouse head and target position. Left panel shows the head and target positions during 'Following'. 'Following' is defined as a period in which the head is close to the target $(<15 \mathrm{~mm} x$-axis, $<10 \mathrm{~mm}$ in $y$-axis, $<20 \mathrm{~mm}$ in $z$-axis). Right panel shows the head and target positions during 'Not following'. Also see Supplementary movies 1 and 2 for examples. c Average distance is significantly reduced during following (paired $t$-test, $p<$ 0.0001). Coefficient of variation (CV) in distance shows that variance is also increased when animals are not following. $\mathbf{d}$ Average self-velocity is significantly lower during following (paired $t$-test, $p<0.0001$ ). CV in velocity is also increased when animals are not following. e Illustration of the temporal relationship between distance and self-velocity. Cross-correlation analysis reveals the lag between these two variables, showing that distance leads selfvelocity during pursuit behavior. In addition, the lag shows a positive correlation with distance $(p<0.0001)$. When the mouse is actively pursuing and maintaining a small distance, the lag between distance and velocity is small, but the lag increases as pursuit performance declines. Error bars indicate \pm s.e.m. ${ }^{\star \star \star} p<0.0001$. Source data are provided as a Source Data file

in $\mathrm{PV}+$ neurons $(\mathrm{PV}-\mathrm{Cre})^{21}$. Only neurons that are activated by light stimulation with a short latency $(<8 \mathrm{~ms})$ are classified as FSIs (Supplementary Table 5). The optogenetically tagged cells show similar waveforms and firing rates as those we classified as
FSIs (Fig. 4a, b). More importantly, most identified FSIs are also correlated with distance to target (Fig. 4c-e). The population average activity shows a highly linear relationship with distance (Fig. 4f). 
a

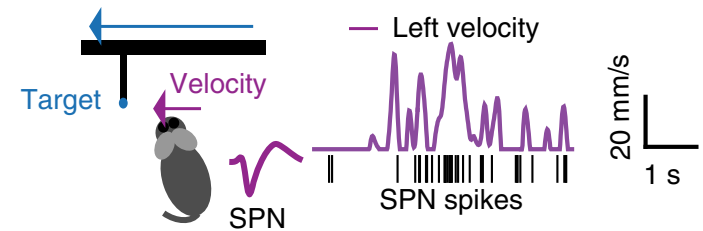

b
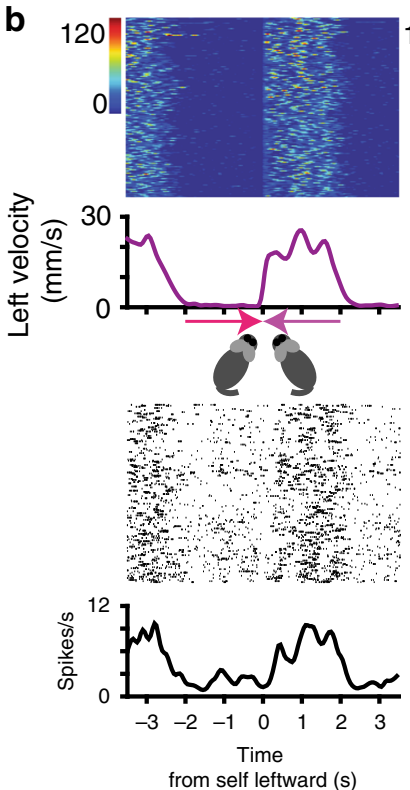

C

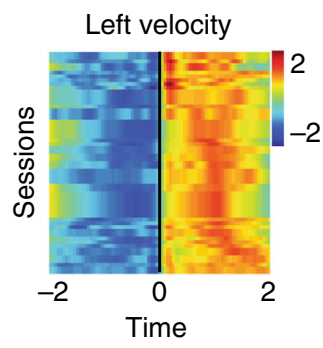

from self leftward (s)

g

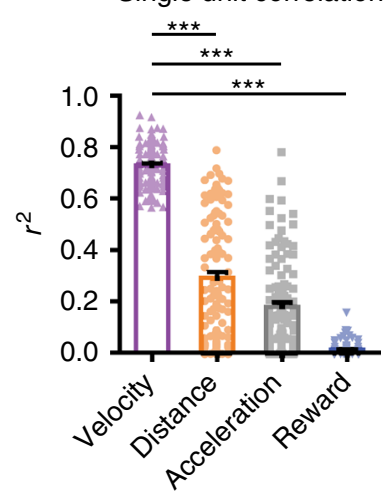

from self leftward (s)

h d

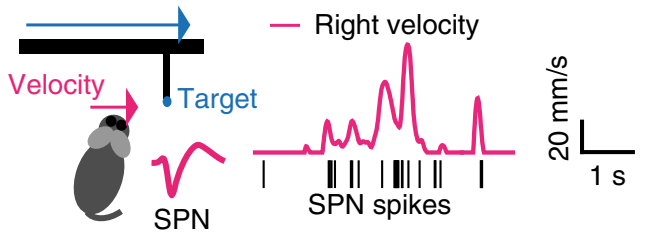

e
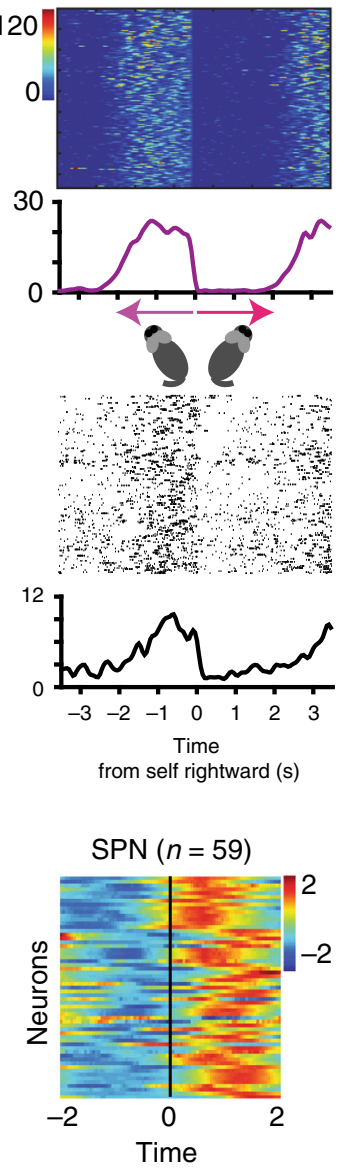

Following

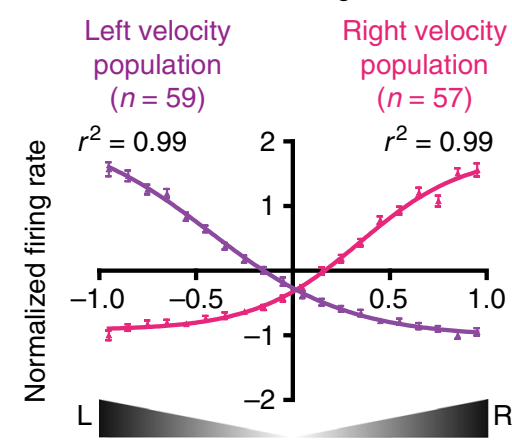

Normalized velocity
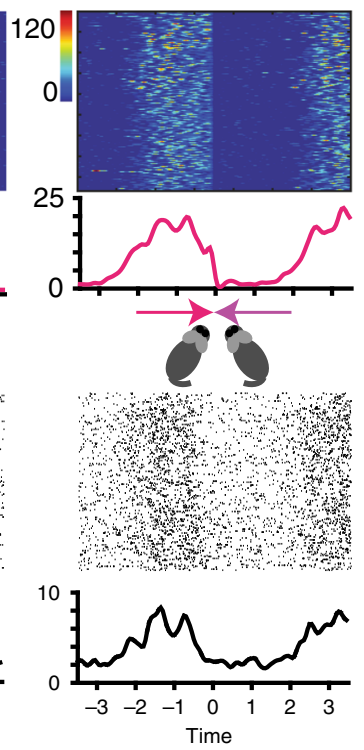

from self leftward (s)

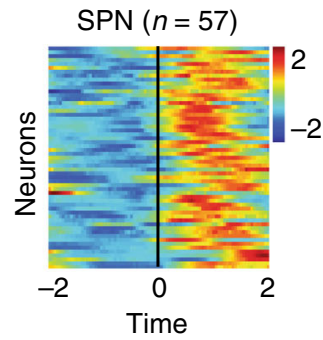

from self rightward (s) from self rightward (s)

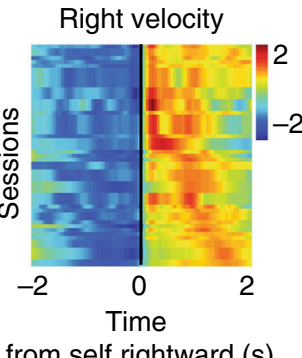

i
Not following

$$
\begin{array}{cc}
\text { Left velocity } & \text { Right velocity } \\
\text { population } & \text { population } \\
(n=59) & (n=57)
\end{array}
$$

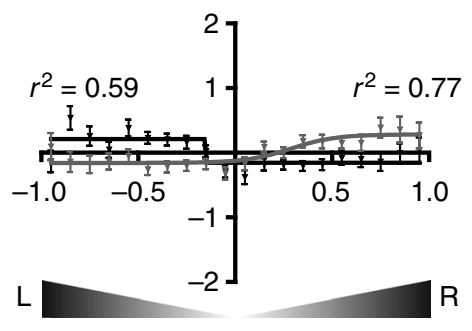

Normalized velocity
Finally, to confirm our in vivo electrophysiological results, we also utilized calcium imaging with miniaturized endoscopes to record FSI activity (Fig. 4g, h, Supplementary Tables 6 and 7) 22,23 . In agreement with our electrophysiological data, we found many FSIs whose activity represents distance (Fig. 4i-k). Population average activity of FSIs also showed a linear relationship with distance to target, with two opponent populations (Fig. 4l).
Manipulations of neural activity. Our electrophysiological and imaging results show that distance to target, the key variable in pursuit behavior, is represented by striatal FSI neurons. However, they do not tell us whether this representation is required for successful pursuit. To test necessity, we silenced FSIs using the tetanus toxin light chain (TeLC). TeLC prevents neurotransmitter release by cleaving its vesicle-associated membrane protein $2^{24,25}$. We used Cre-dependent TeLC (AAV-flex-TeLC) in PV-Cre mice 
Fig. 2 SPNs represent self-velocity when animal is following target. a A representative SPN with spike waveform. The firing rate of this neuron is positively correlated with leftward velocity. b Raster plots comparing single unit activity of a representative left velocity SPN and velocity. Time zero is the start of leftward or rightward pursuit behavior. Note the similarity between velocity and firing rate. c Spike density function of all left velocity SPNs ( $z$-scored values). Each row represents average velocity measure and the firing rate of corresponding SPNs from a single session. Note that some rows in the velocity plot are identical, because multiple neurons from the same mouse are correlated with the same velocity variable. d A representative SPN with spike waveform. The firing rate of this neuron is positively correlated with rightward velocity. e Raster plots comparing velocity and firing rates of a right velocity SPN. f Spike density function of all right velocity SPNs ( $z$-scored values). $\mathbf{g}$ The correlation coefficient between firing rate of individual velocity SPNs and different behavioral variables. In each case, velocity has the highest $r^{2}$ value (repeated measures ANOVA: main effect of variable, $F_{3,463}=453.8, p<$ 0.0001; Bonferroni post hoc: velocity vs. distance $p<0.0001$, velocity vs. acceleration $p<0.0001$, velocity vs. reward $p<0.0001$ ). Error bars indicate \pm s.e.m. ${ }^{\star \star \star} p<0.0001$. h Population average activity of all velocity-correlated SPNs. $\mathbf{i}$ Velocity SPNs show much weaker correlation if the mouse is not following. Source data are provided as a Source Data file

(Fig. 5a), thus expressing TeLC in a cell type-specific and regionspecific manner (Fig. 5b, Supplementary Fig. 6). TeLC was injected after training, and we tested the mice again following recovery. FSI silencing markedly disrupted target pursuit performance, without affecting overall movement velocity during the session (Fig. 5c-e).

TeLC effects are permanent, making it difficult to rule out compensatory plasticity due to reduced FSI synaptic transmission. We therefore used designer receptors exclusively activated by designer drugs (DREADDs) to suppress FSI activity reversibly (Fig. $5 \mathrm{f})^{26}$. We injected an AAV vector encoding a Cre-dependent designer G protein-coupled hM4Di receptor bilaterally (Fig. 5g, Supplementary Fig. 7). Injection of the synthetic ligand clozapine$\mathrm{N}$-oxide (CNO) for the hM4Di receptor reduced the amount of time spent following the target (Fig. 5h) and increase distance to target (Fig. 5i) without affecting movement velocity in general (Fig. 5j).

To test the effects of temporally specific manipulation of FSI activity, we performed optogenetic experiments to manipulate FSI activity bidirectionally during behavior ${ }^{21,27}$. FSIs were excited using the excitatory channelrhodopsin (ChR2) injected in the sensorimotor striatum of PV-Cre mice (Fig. 6a, Supplementary Fig. 8) and inhibited using the inhibitory channelrhodopsin stGtACR2, which conducts anions (both can be activated by blue light, Fig. 6b, Supplementary Fig. 9) ${ }^{28-30}$. As expected, bilateral ChR2 activation of FSIs increased distance to target, whereas bilateral stGtACR2 inhibition of FSIs decreased distance (Fig. 6c, Supplementary Fig. 10). Since FSIs are known to provide inhibition of SPNs, these manipulations have the opposite effects on velocity (Fig. 6d).

Working model. Both SPNs and FSIs are known to receive convergent inputs from different cortical regions ${ }^{31,32}$. We hypothesize that these inputs can provide information on distance to target. Based on the anatomy of the FSI-SPN circuit and our results, we propose a working model for how this circuit contributes to pursuit behavior (Fig. 7). According to this model, the SPNs and FSIs that form a functional unit share excitatory cortical inputs. Signals representing target distance are sent via the corticostriatal projections to a subset of FSIs and SPNs. FSI output sends a slightly delayed of this signal to the relevant SPNs. Consequently, a given velocity SPN can receive two inputs, one net positive signal from the cortex that represents distance, and a slightly delayed version of the same input, with the sign inverted, from the FSI. Because the FSI-SPN projection is inhibitory (Supplementary Fig. 11), it is subtracted from the excitatory input, and the SPN output will then reflect the difference between excitatory cortical input and a temporally delayed version of the same signal. This 'temporal difference' signal, similar to taking the time derivative of the distance representation, is then used as a velocity command to drive performance. At rest, velocity is close to zero, consistent with the typically low baseline firing rates of SPNs ${ }^{33}$. By contrast, FSIs are characterized by high tonic firing rates, which make them more suitable for signaling the distance variable. Tonic FSI firing close to the middle of the range is used to represent the effective zero, when the target is directly in front of the mouse. Different types of errors in distance control (left vs. right) are indicated by the sign of the signal relative to the effective zero (Fig. 3) 34,35 . For neurons that increase firing when the target is to the left, the left extreme is represented by the highest firing rate and the right extreme is represented by the lowest firing rate, and vice versa for neurons that increase firing when target is on the right side. A positive error means that distance error has increased, i.e. the target is farther away in a particular direction, and velocity in that direction must be increased accordingly to reduce the distance error. Thus an increase in left distance increases the temporal difference signal computed by the FSI-SPN circuit, and a corresponding increase in the leftward velocity command. A negative difference signal indicates that the distance error is decreasing and velocity in that direction should be reduced. SPN output is therefore regulated by the change in distance between self and target in real time.

Our working model is supported by optogenetic manipulation of SPNs. We used SIO-GtACR to inhibit SPNs in the direct $(\mathrm{D} 1+)$ and indirect $(\mathrm{A} 2 \mathrm{~A}+)$ pathways (Supplementary Fig. 12). Inhibition of SPNs in either pathway did not significantly change distance to target. However, direct pathway inhibition reduced velocity and increased distance error, whereas indirect pathway inhibition slightly increased velocity. These results suggest that direct pathway SPNs are responsible for the velocity commands.

\section{Discussion}

To successfully pursue a moving target, it is necessary not only to regulate velocity and direction of movement but also to continuously monitor the distance between self and target. Here we showed for the first time that mice use internal representations of distance to guide their pursuit behavior (Fig. 1). We also found that a striatal microcircuit is critical for pursuit behavior. Striatal FSIs not only represent target distance, and are also necessary for successful pursuit performance. Their projections to the downstream SPNs are critical in regulating self-velocity during pursuit.

In our task, the target always moves from side to side, each cycle consisting of both leftward and rightward movements. At any time, the target could be on either side of the mouse. We identified distinct types of self-target distance representations in FSIs. Some neurons increase firing when the target moves to the left side of the animal, and decrease firing when it moves to the right, and vice versa for other neurons (Fig. 3). On the other hand, SPNs are strongly correlated with movement velocity, with clear direction-specificity (Fig. 2).

In order to test whether FSIs are necessary for pursuit, we used a variety of methods to manipulate their activity. Silencing these neurons using hM4Di or TeLC resulted in impaired pursuit (Fig. 5). However, as these techniques lacked temporal precision, 
a
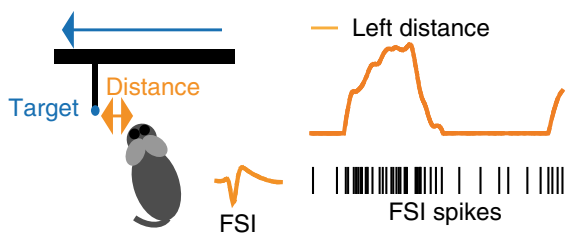
$\underset{\text { | }}{|||||||||||||||||||||||||||| \mid}$

b
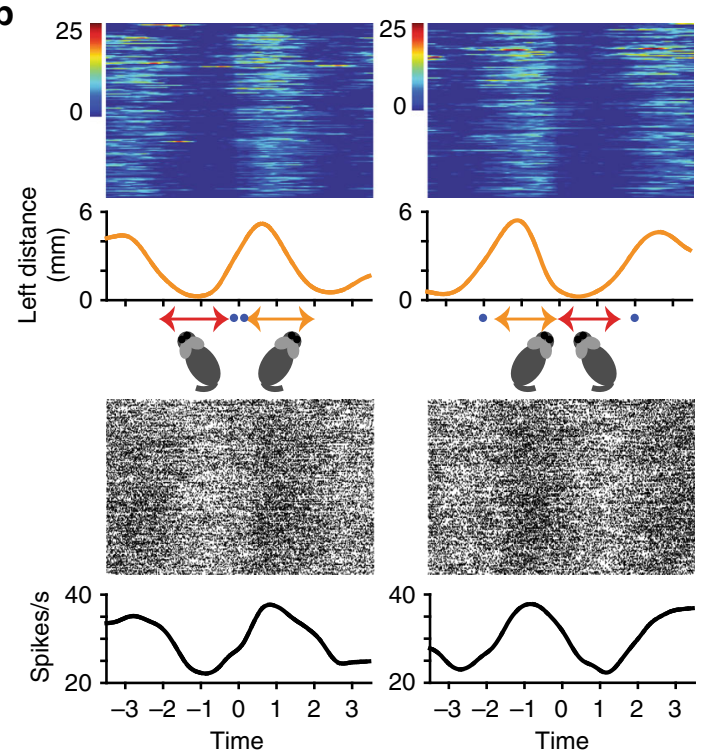

from self leftward (s)

C

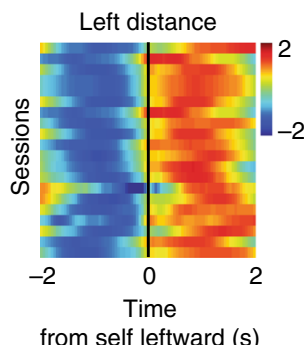

9
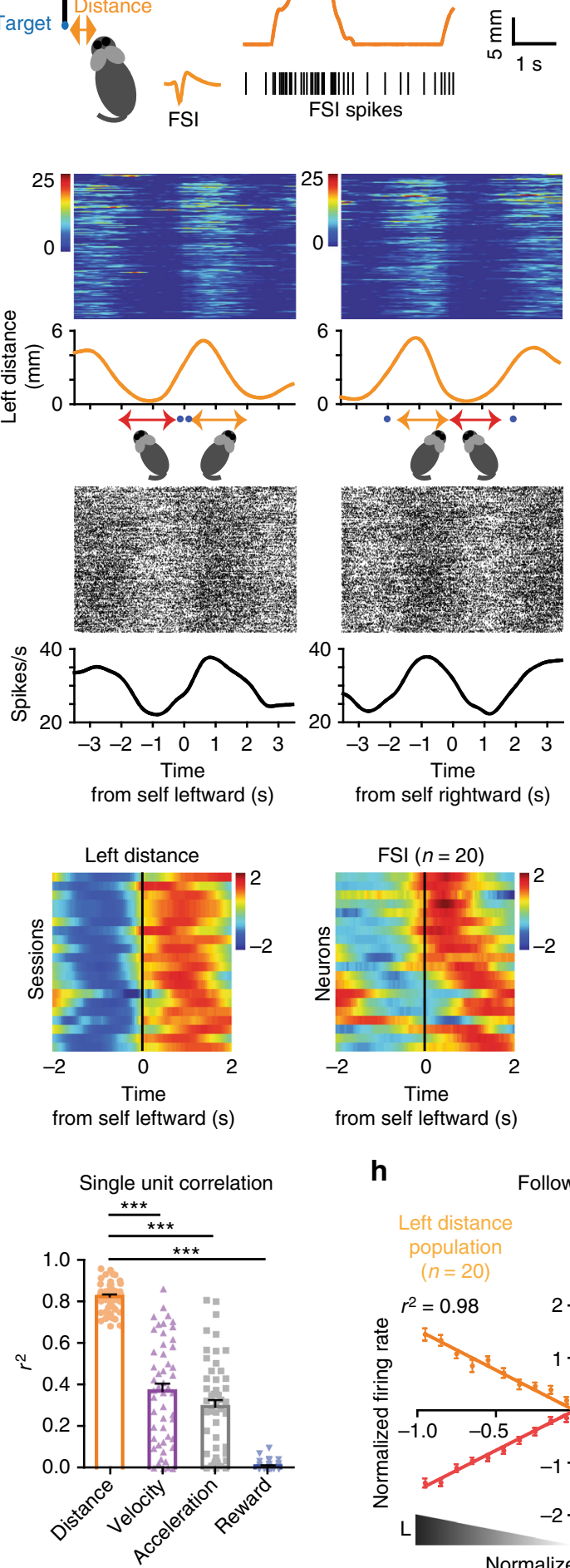

from self leftward (s)

$\mathbf{h}$ d

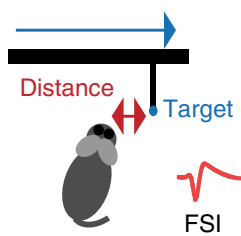

- Right distance

e
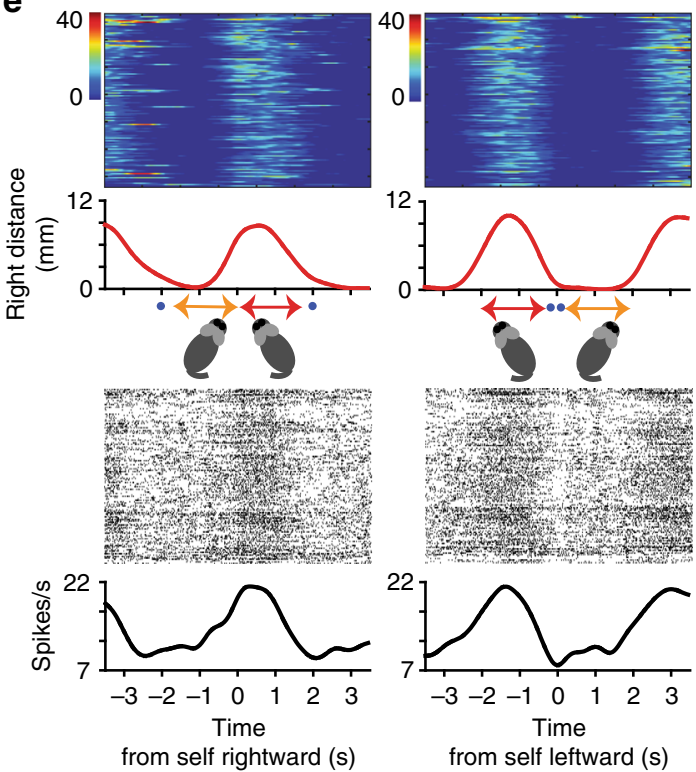

$\mathbf{f}$

Following

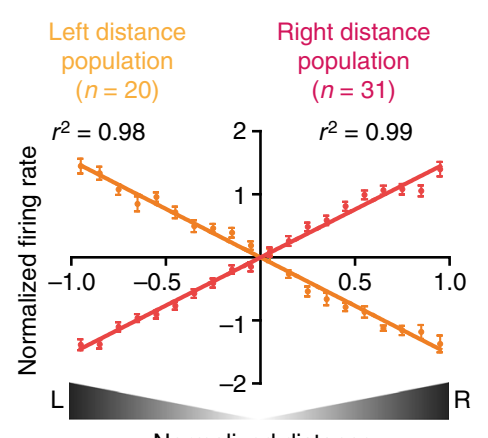

Normalized distance

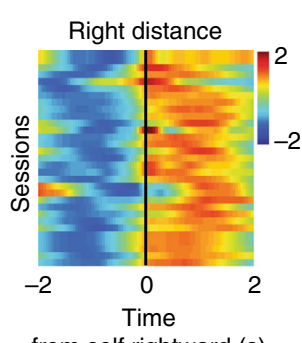

from self rightward (s)
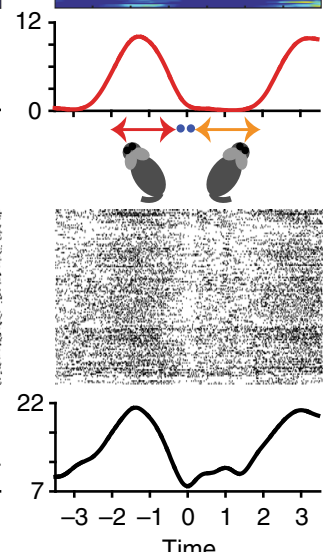

from self leftward (s)

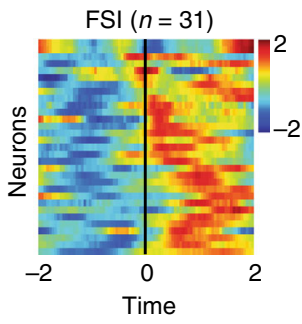

from self rightward (s) i

Not following

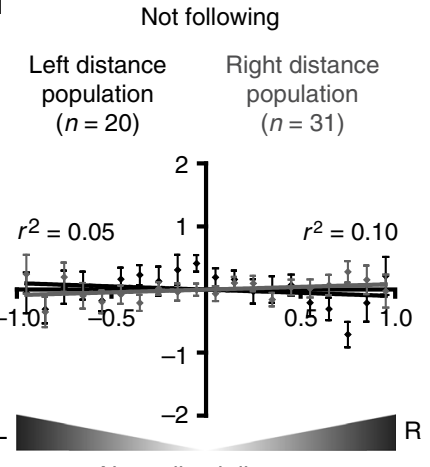

Normalized distance it was only possible to measure overall distance and velocity during the session. It was not possible to assess the immediate impact of FSI silencing on behavior. Using optogenetics, we were able to obtain a better understanding of how bidirectional manipulation of FSI activity affected pursuit performance. In particular, inhibition using GtACR2 reduced distance while excitation using ChR2 increased distance. As expected, these manipulations have the opposite effects on velocity given the mainly inhibitory effects of FSIs on SPNs (Fig. 6). While these effects support the hypothesis that FSIs are critical for pursuit, it is unclear whether sensory detection in general was affected by manipulation of FSI activity. That is, we cannot easily rule out the possibility that FSIs are also critical for distance perception per se. The role of striatal FSIs in sensory processing remains to be elucidated.

Recent studies have also elucidated neural substrates underlying natural prey pursuit in hunting behavior. A key area that has been reported to be important for prey pursuit is the 
Fig. $3 \mathrm{FSIs}$ can represent distance to target. a A representative FSI with spike waveform. The firing rate of this neuron is positively correlated with distance to target on the left side of the head, regardless of whether the mouse is moving leftward or rightward. $\mathbf{b}$ Raster plots comparing single unit activity of a representative left distance FSI and distance. Time zero is the start of leftward or rightward pursuit. c Spike density function of all left distance FSIs. Each row represents the average distance measure and the firing rate measure of the corresponding FSI from a single session. Note that some rows in the velocity plot are identical, because multiple neurons are correlated with the same distance variable. d A representative FSI with spike waveform. The firing rate of this neuron is correlated with distance to target on the right side of the head, regardless of whether the mouse is moving leftward or rightward. e Raster plots comparing single unit activity of a representative right distance FSI and distance. Time zero is the start of leftward or rightward pursuit. f Spike density function of all right distance FSIs. $\mathbf{g}$ The correlation coefficients between firing rate of distance FSIs and alternative behavioral variables. In each case, distance has the highest correlation with firing rate (repeated one-way ANOVA $F_{3,203}=193.0, p<0.0001$; Bonferroni post-hoc: distance vs. velocity $p<0.0001$, distance vs. acceleration $p<0.0001$, distance vs. reward $p<0.0001$ ). Error bars indicate \pm s.e.m. ${ }^{\star \star \star} p<0.0001$. $\mathbf{h}$ Population average activity of all distance-correlated FSIs. i Distance FSIs show much weaker correlation when not following. Source data are provided as a Source Data file

periaqueductal gray (PAG). This area is the target of descending projections from many regions, and recent work has shown that projections from the central amygdala and hypothalamus can activate pursuit behavior ${ }^{36-38}$. Interestingly, PAG also receives inputs from the $\mathrm{BG}^{39}$. It is therefore possible that the $\mathrm{BG}$ are one source of descending projections to the PAG that can also guide pursuit behavior.

Our results suggest that tonic FSI firing close to the middle of the dynamic range is used to represent the effective zero in horizontal distance, when the target is directly in front of the mouse. Different types of errors (left vs. right) are indicated by the sign of the signal in relation to the effective zero. For example, for neurons that increase firing when the target is to the left, the left extreme corresponds to the highest firing rate and the right extreme to the lowest firing rate, and vice versa for neurons that increase firing when the target is on the right side. These two types of FSIs may project to distinct types of SPNs that signal velocity. A distance error signaling 'too much to the left' would suppress rightward neurons and activate leftward SPNs, whereas 'too much to the right' would suppress leftward neurons and activate rightward SPNs. This organization would enable bidirectional control by FSIs.

In our working model, we assume that the distance FSIs and velocity SPNs share cortical inputs, which represent distance to target. Yet the circuit computation generates instantaneous velocity commands from SPNs, because the feedforward FSI circuit allows differentiation of the distance variable during tracking. Previous optogenetic experiments showed that movement velocity depended on the frequency of striatonigral stimulation ${ }^{19}$. We also showed that inhibition of the direct pathway $(\mathrm{D} 1+)$ neurons significantly reduced velocity during pursuit, but inhibition of indirect pathway $(\mathrm{A} 2 \mathrm{~A}+)$ neurons did not. Thus, the distance to velocity conversion is achieved mainly by the direct pathway. Previous work also showed that GABAergic output from the substantia nigra pars reticulata, the main output of the direct pathway, represents instantaneous position vector components ${ }^{34}$. Using the striatonigral pathway, velocity commands could be integrated into reference signals representing proprioceptive position references that change over time $34,35,40-43$. These reference signals from the BG output would then reach downstream proprioceptive position control systems for posture and body configuration $^{44,45}$.

Although velocity and distance are both continuous, timevarying variables, there are key differences between them. Movement velocity, detectable by mainly proprioceptive and vestibular sensory inputs ${ }^{40}$, is close to zero at rest. The usually low firing rates of SPNs are ideal for representing this type of variable $^{33}$. On the other hand, distance, usually detected by teloreceptive inputs, is rarely close to zero. FSIs are characterized by high tonic firing rates, which make them ideal for signaling distance. Presumably inputs from sensory cortical areas provide distance information that is sent to the striatal FSIs. Visual inputs are obviously critical, but we cannot rule out other sensory modalities. Based on our results, we hypothesize that representations of specific pursuit errors can activate the appropriate combination of direction-specific SPNs (e.g. moving leftward when the target is on the left side) and suppress the conflicting SPNs. With their strong inhibitory projections to SPNs, FSIs can suppress the activation of irrelevant or antagonistic SPNs via monosynaptic inhibition. On the other hand, via disinhibition or perhaps excitatory effects of $\mathrm{GABA}^{46}$, they can also have net excitatory effects on downstream SPNs.

Given the importance of approaching a spatially discrete target in normal behavior, it is not surprising that striatal FSIs have been implicated in so many functions ${ }^{14-16}$. For example, although reward is the eventual goal of behavior in our task, the striatal neurons we recorded represented specific spatial variables necessary for successful performance, rather than reward prediction or consumption. In accord with previous work ${ }^{17}$, we found that, in the sensorimotor striatum at least, FSI and SPN activity is largely independent of reward delivery. Although some studies have suggested that striatal neurons are modulated by reward, they did not monitor continuous behavioral variables ${ }^{47}$. Standard manipulations like reward size or probability may also alter distance or velocity variables. It is also impossible to explain our results in terms of predicted reward value because FSI activity reflects distinct distance errors that are spatially defined, e.g. left and right. Extreme caution is therefore needed in interpreting experiments that lack precise and continuous behavioral measures. Abstract psychological concepts like reward value must be well anchored to clear experimental measures to generate interpretable results.

Our results also resolve current controversies on the relationship between striatal activity and behavior. A recent study by Owen et al. concluded that FSIs are not needed for behavioral performance but for learning and plasticity ${ }^{48}$. But they did not use continuous behavioral measures and consequently failed to detect the crucial relationship between FSI activity and key performance variables like distance to target. In fact, because their learning tasks involved orienting and approaching reward targets, any learning defects observed following disruption of FSI output can be explained by performance deficits in approaching the target. Moreover, while some recent studies observed correlation between velocity and striatal output ${ }^{18,49}$, this conclusion has also been questioned. For example, Klaus et al. argued that SPNs can encode action identity independent of velocity ${ }^{50}$, but their conclusion is not supported by the data, because their accelerometer measures lack spatial resolution and their calcium imaging results lack temporal resolution. Thus, they were not able to measure velocity vector components and quantify their relationship with neural activity. Moreover, free behavior in an open field is exceedingly complex and not always dependent on striatal output, so caution is also needed in interpreting neural activity recorded during such behavior. 
a

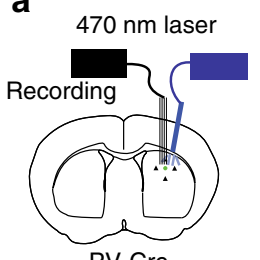

b
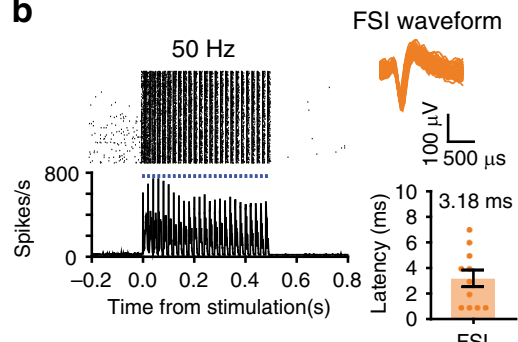

g

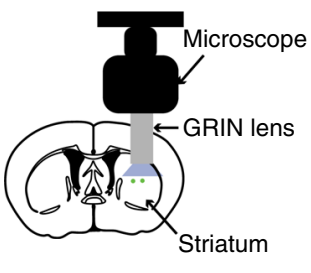

h
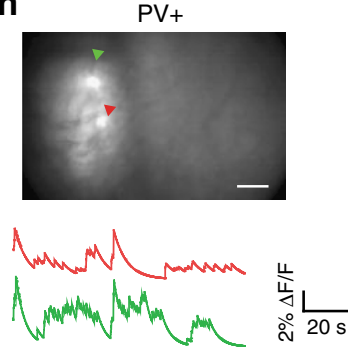

i
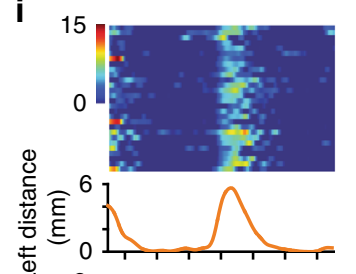

$$
\text { 零 }
$$

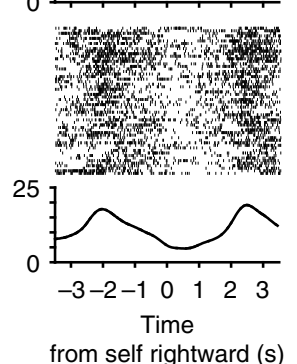

from self leftward (s)

from self rightward (s)

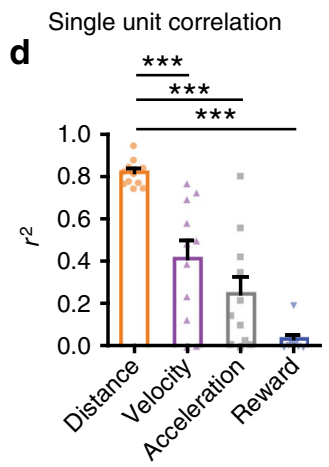

e

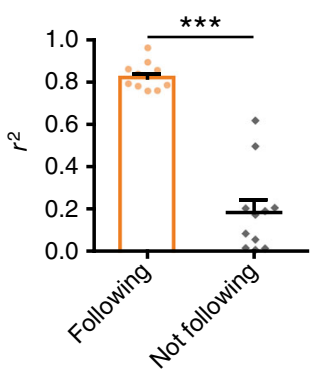

$\mathbf{f}$

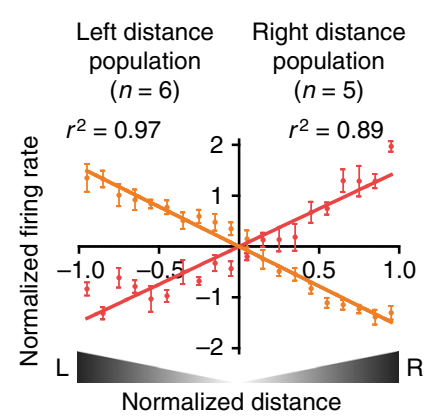

On the other hand, while classic studies discovered SPNs linked to movement speed $^{51,52}$, they did not measure behavior continuously in freely moving animals and relied on average measures. Kim et al. were the first to show that SPNs can represent vector components of velocity and to demonstrate that such representations are similar whether or not the behavioral outcome is rewarding or aversive ${ }^{17}$. Just like a recent study using calcium imaging in head restrained mice ${ }^{53}$, Kim et al. concluded that both SPNs and FSIs represented velocity. However, the
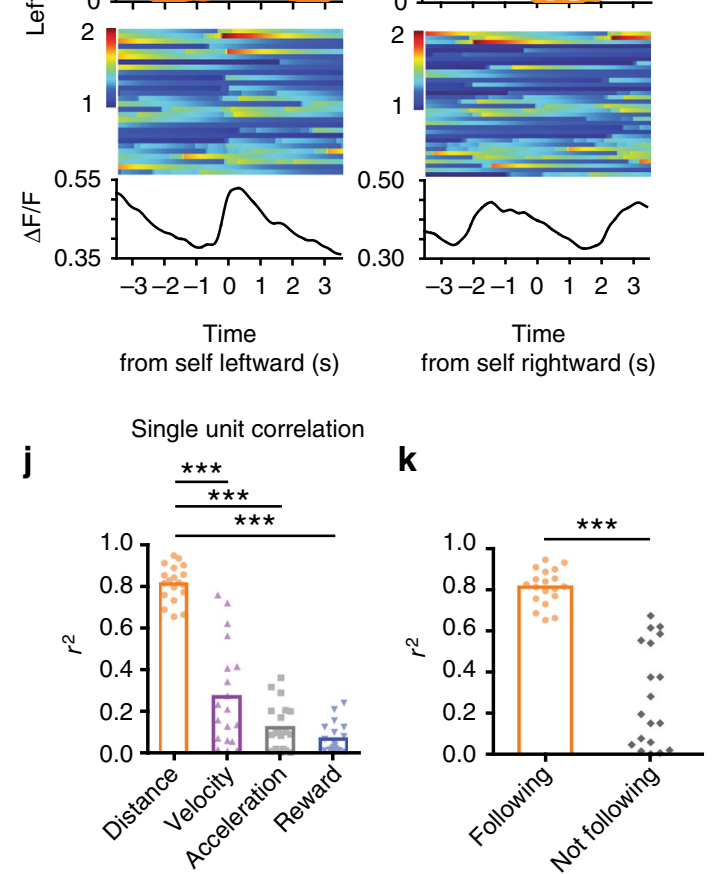

I

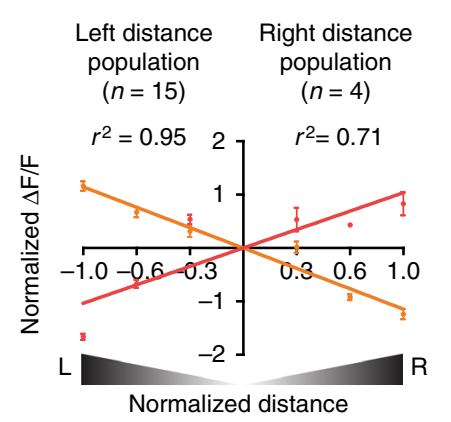

video-based motion tracking used in their study did not have sufficient spatial and temporal resolution to dissociate the different contributions of SPNs and FSIs. In the present study the use of 3D motion capture reveals that, during tracking, FSIs more commonly represent distance to target, while many sensorimotor SPNs represent velocity.

The velocity representation in a large proportion of sensorimotor SPNs is therefore unique, and so far not found in any other cell type except nigrostriatal dopamine neurons ${ }^{41}$. Striatal 
Fig. 4 Optotagging single unit activity and in vivo calcium imaging of FSI activity during pursuit. a Schematic illustration of simultaneous optogenetic stimulation and recording using a chronically implanted optrode. An AAV vector with Cre-inducible ChR2 is injected into the sensorimotor striatum of PVCre mice to allow selective activation of PV+ FSIs. b A representative left distance neuron that is optically tagged. ChR2-induced stimulation at $50 \mathrm{~Hz}$ reliably triggered spiking in FSIs with a short latency (average latency $=3.18 \pm 0.65 \mathrm{~ms}$ ). c Representative left distance FSI that is optically tagged. The firing rate of this neuron is proportional to leftward distance. Time zero is the start of leftward or rightward pursuit. $\mathbf{d}$ Correlation between optotagged distance FSIs and different behavioral variables (repeated one-way ANOVA $F_{5,43}=30.98, p<0.0001$; Bonferroni post-hoc: distance vs. velocity $p<0.0001$, distance vs. acceleration $p<0.0001$, distance vs. reward $p<0.0001)$. ${ }^{\star \star \star} p<0.0001$. e Correlation between FSI distance neurons and distance is significantly lower when the animal is not following the target (paired-t test, $p<0.0001$ ). f Population summary of all optotagged distance FSI neurons. $\mathbf{g}$ Calcium imaging with an implantable GRIN lens $(1.8 \mathrm{~mm}$ diameter). Schematic illustration of the GRIN lens implant. A Cre-dependent GCamp6s virus was injected into the sensorimotor striatum of PV-Cre mice. $\mathbf{h}$ Representative image of FSIs. Red and green traces indicate two simultaneously imaged FSIs. Because FSIs are rare, only 2-11 cells were recorded per mouse. Also see Supplementary movie 3. i Representative left distance FSI. Calcium signal from this neuron is proportional to leftward distance. Time zero is the start of leftward or rightward pursuit. $\mathbf{j}$ Correlation between distance FSIs and different behavioral variables (repeated one-way ANOVA, $F_{3,75}=103.4, p<0.0001$; Bonferroni post hoc analysis: distance vs. velocity $p<0.0001$; distance vs. acceleration $p<0.0001$; distance vs. reward $p<0.0001$ ). $\mathbf{k}$ Correlation between FSI distance neurons and distance is significantly lower when the animal is not actively following (paired $t$-test, $p<0.0001$ ). I Population summary of all distance FSIs from in vivo calcium imaging. Error bars indicate \pm s.e.m. Source data are provided as a Source Data file
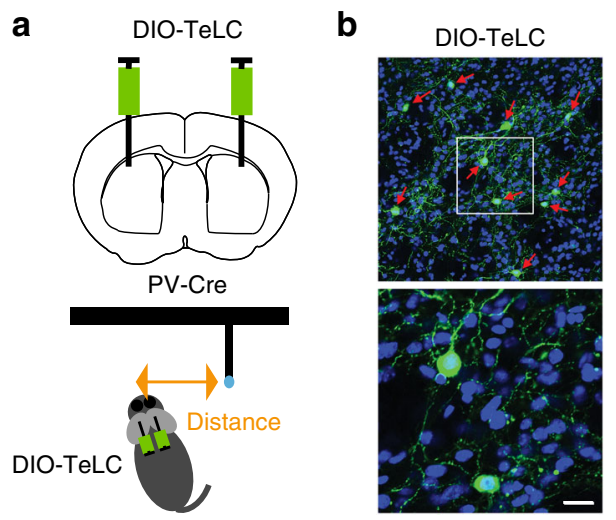

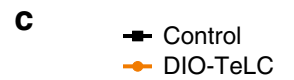

d
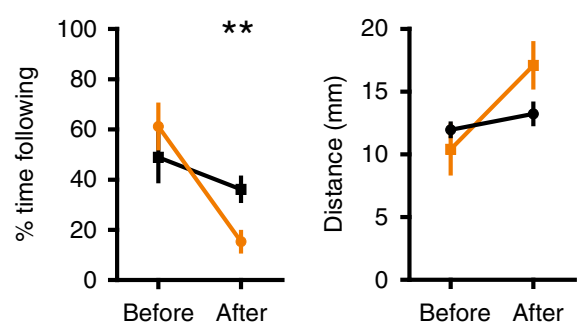

f

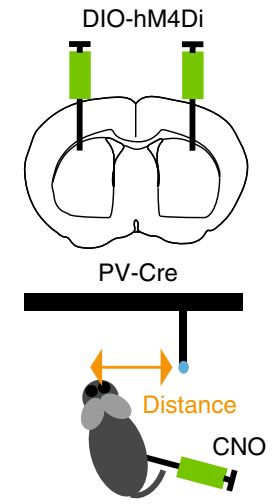

g

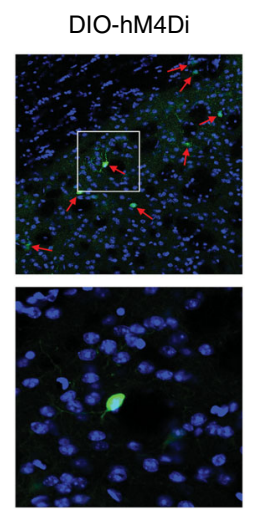

h

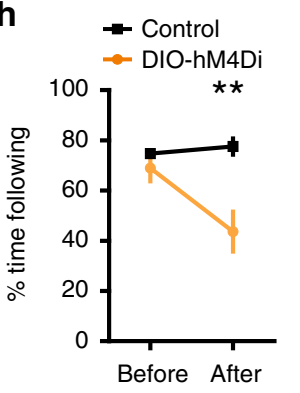

i

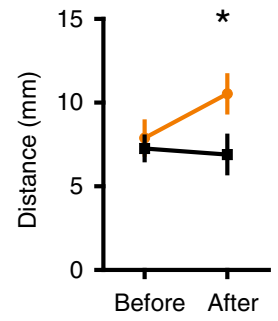

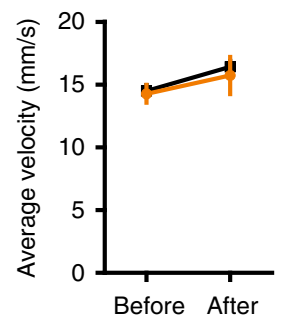

e
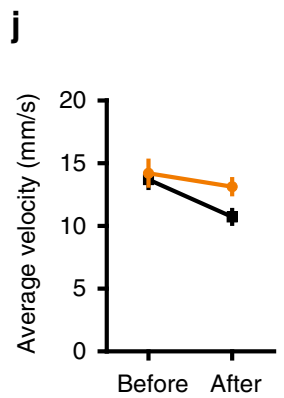

Fig. 5 Silencing FSI activity disrupts pursuit performance. a To silence neural activity in FSIs, we injected the Cre-dependent DIO-TeLC virus into the striatum of PV-Cre mice. TeLC silences neurons by cleaving the vesicular-associated membrane protein 2 and preventing transmitter release. $\mathbf{b}$ GFP staining showing FSIs infected with GFP-tagged TeLC (control: $n=6$, DIO-TeLC: $n=7$ ). c Duration of self-initiated pursuit behavior (\% time spent following) is significantly reduced by TeLC. A repeated measures ANOVA revealed a significant interaction: $F_{1,11}=6.88, p=0.024$. Post hoc test showed a significant effect of TeLC $(p=0.0051)$. ${ }^{\star *} p<0.01$. d Distance error is greater in the TeLC group compared to the Control group (GFP only). Repeated measure ANOVA: Interaction: $F_{1,11}=5.92, p<0.05$. Post hoc unpaired $t$-test, $p=0.09$. e TeLC did not affect velocity: no significant interaction: $F_{1,11}=$ $0.08, p=0.77$, no effect of Time, $F_{1,11}=12.73, p=0.05$, or of Group: $F_{1,11}=0.94, p=0.67$. f To reversibly inactivate FSIs, we injected the Cre-dependent DIO-hM4Di virus into the striatum. $\mathbf{g}$ GFP staining showing FSIs in the striatum infected with GFP-tagged DREAAD (control: $n=6$, DIO-hM4Di: $n=6$ ). h hM4Di reduced \% time spent following the target. There was a significant interaction: $F_{1,10}=15.2, p=0.0030$. Post hoc test revealed a significant effect of CNO after injection $(p=0.0028) .{ }^{\star \star} p<0.01$. i hM4Di increased distance: Interaction: $F_{1,10}=9.26, P=0.044 .{ }^{*} p<0.05$. j hM4Di did not affect velocity. No interaction: $F_{1,10}=5.11, p=0.12$, main effect of time: $F_{1,10}=23.21, p<0.01$, no effect of Group: $F_{1,10}=12.09, p=0.11$ ). Error bar indicates \pm s.e.m. Source data are provided as a Source Data file

velocity neurons have strong preference for direction of egocentric motion (left, right, up, down). Actual volitional movement is shaped by a combination of distinct vector components (at least four major classes depending on the direction of movement). A population of such velocity-related neurons can therefore represent a velocity vector.

In addition, although previous work found that SPNs are typically selective for contraversive movements ${ }^{54}$, we did not find 
a

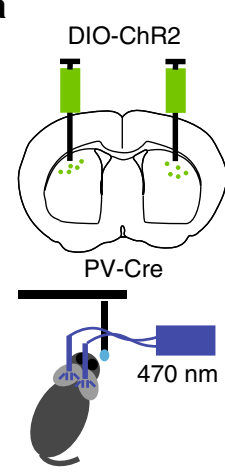

C

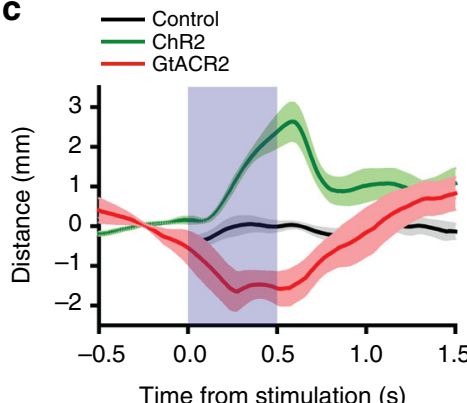

Excitation of FSI
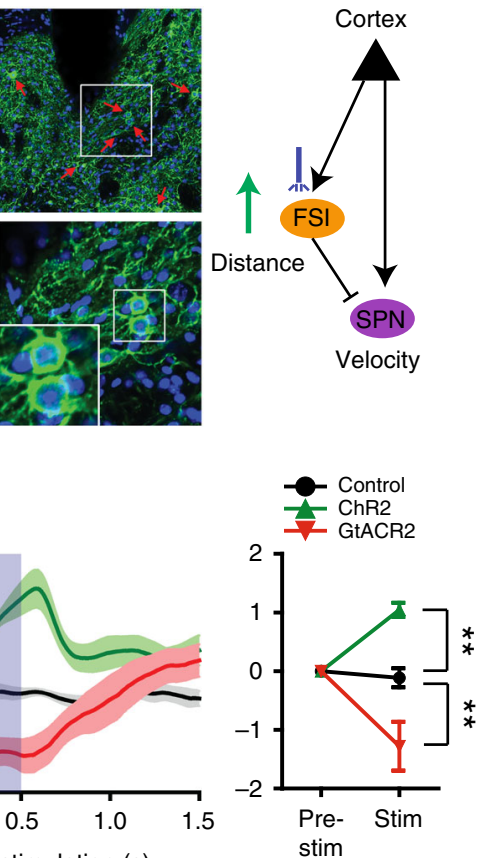

b

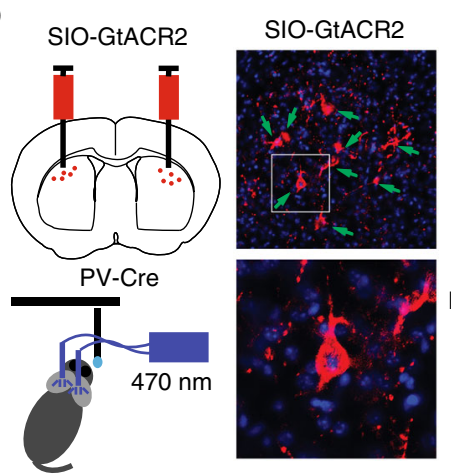

d

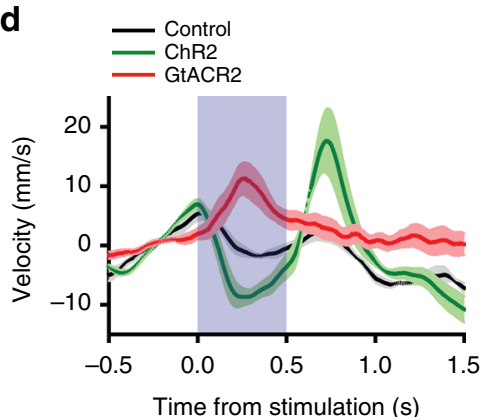

Inbition of FSI
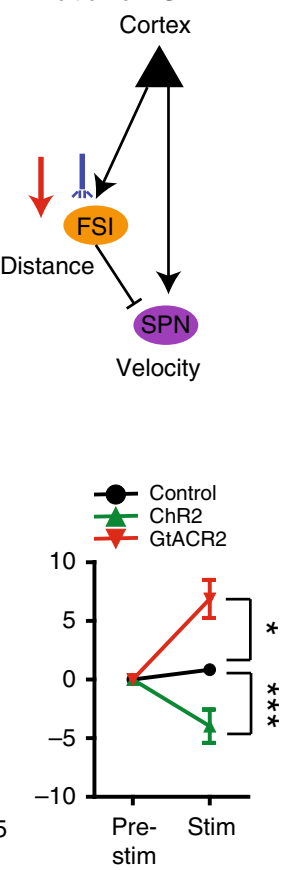

Fig. 6 Optogenetic manipulation of FSI activity disrupts pursuit performance. a We injected Cre-dependent excitatory channelrhodopsin (AAV-EF1a-DIOChR2-EYFP) into the sensorimotor striatum of PV-Cre mice. Middle, GFP staining showing FSIs infected with ChR2. This manipulation is expected to increase FSI activity and reduce target SPN activity. b We injected Cre-dependent inhibitory channelrhodopsin (AAV-hsyn1-SIO-GtACR2-Fusion Red) to reduce FSI activity and increase target SPN activity via disinhibition. c Effect of photo-stimulation on self-target distance. Increasing FSI activity with ChR2 increased distance, whereas decreasing FSI activity with GtACR2 decreased distance (repeated measures two-way ANOVA, Interaction: $F_{2,11}=12.68, p<$ 0.0014, Group: $F_{2,11}=12.68, p=0.0014$; Time: $F_{1,11}=0.35, p=0.57$, Boferroni post-hoc: control vs. ChR2, $p<0.01$; control vs. GtACR2, $p<0.01$ ). PV-Cre control: $n=4$; ChR2: $n=4$; GtACR2: $n=6$. ${ }^{\star \star} p<0.01$. d Effect of photo-stimulation on self-velocity. Since FSls inhibit SPNs, the effects on velocity are expected to be the opposite as those on distance in c. ChR2 decreased velocity, whereas GtACR2 increased velocity (repeated measures two-way ANOVA, Interaction: $F_{2,11}=15.16, p<0.0007$, Group: $F_{2,11}=15.16, p<0.0007$; Time: $F_{1,11}=2.17, p=0.17$, control vs. ChR2, $p<0.05$; control vs. GtACR2, $p<$ 0.001). Error bar indicates \pm s.e.m. ${ }^{\star \star \star} p<0.001$. ${ }^{\star} p<0.05$. Source data are provided as a Source Data file

more contraversive neurons than ipsiversive neurons. There are two possible explanations for this discrepancy. First, we might not have sampled enough neurons to obtain an accurate estimate of the distribution of contraversive and ipsiversive neurons. Secondly, the velocity-related SPNs recorded in this study might not be the same as those involved in spontaneous behavior in the absence of an explicit target. While these neuronal populations could overlap, additional neurons that receive bilateral projections from the cortex may be recruited for the pursuit task.

A striking feature of our results is the high correlations between single unit activity and behavioral variables. High correlation is crucial if a signal like firing rate is used in analog computing, as is the case in our model. The concept of correlation is often misunderstood, in part because traditional studies have consistently failed to find high correlations between neural activity and behavior. Low correlation in most studies are responsible for the development of many elaborate statistical techniques and for conflicting interpretations of the neural code $\mathrm{c}^{55}$. For example, multiple regression is often used to analyze single unit data, and many argue that neural signals multiplex multiple behavioral variables, without explaining how to demultiplex the signals. In practice this allows one to argue that any neural signal can multiplex just about any collection of variables. Such a conclusion is hardly falsifiable. To observe the relationship between neural activity and behavior as reported here, it is critical to quantify behavior accurately and continuously in unrestrained animals, and to use measures with high temporal and spatial resolution. Our results suggest that low correlation between neural activity and behavior is due at least in part to limitations in behavioral measures, the widespread use of head restraint, and conventional experimental designs that rely exclusively on categorical classification of behaviors. In contrast, our working model, which involves simple analog computing, has the advantage of being parsimonious and falsifiable.

In conclusion, while our results do not exclude other functional roles for striatal output ${ }^{56}$, they do demonstrate the role of sensorimotor SPNs in the control of velocity during continuous pursuit, and show that the FSI-SPN circuit implements the distance-velocity transformation, the key computation required for the precise guidance of volitional behavior.

\section{Methods}

Animals. Male and female C57BL/6J mice were used in all experiments. For TeLC and optogenetic experiments, Parvalbumin-Cre mice (Pvalb-2A-Cre-D) and D1cre (Drd1a-Cre) and A2A-cre (Adora2a-Cre) mice were used. For DREADD experiments, Drdla-tdTomato:: $P V$-Cre mice were used. Drd1a-tdTomato reporter mice were generated in the Calakos lab (RRID: IMSR_JAX:016204) ${ }^{57}$. All mice were between 3 and 8 months old. They were maintained on a 12:12 light cycle and tested during the light phase. The mice used for electrophysiology experiments were singly housed; others were group housed. During all behavioral experiments, the mice had restricted access to water and food. After each training and recording session, they had free access to water for $\sim 30 \mathrm{~min}$ and $\sim 3 \mathrm{~g}$ of home chow. Their weights were maintained at $85-90 \%$ of their ad libitum weights.

Behavior task and analysis. The moving reward target is a sucrose spout moved by a stepper motor from left to right relative to the mouse standing on a platform (bipolar, $56.3 \times 56.3 \mathrm{~mm}$, DC $1.4 \mathrm{~A}, 2.9 \Omega, 1.8 \%$ step, Oriental motor, USA). The stepper motor was controlled using MATLAB (Mathworks). Two infrared 
a

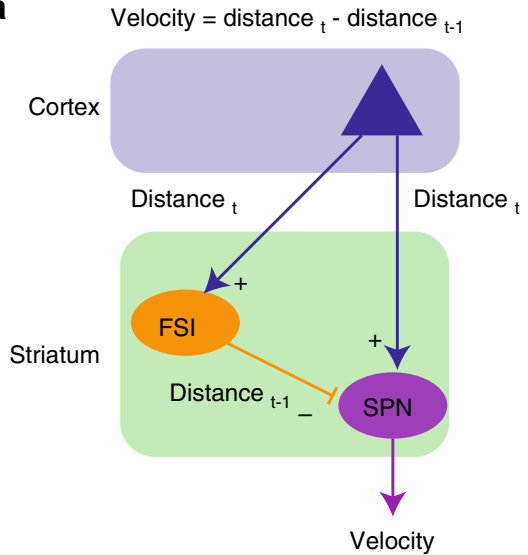

C

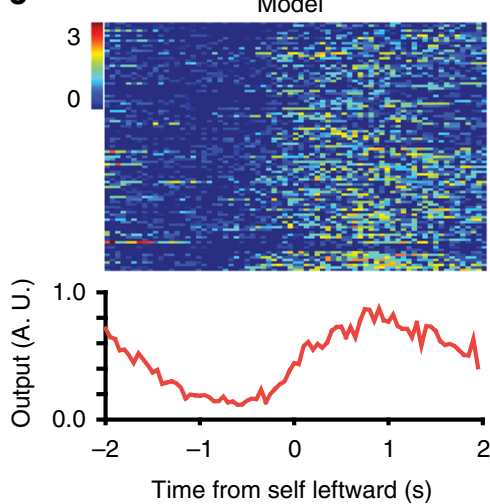

e
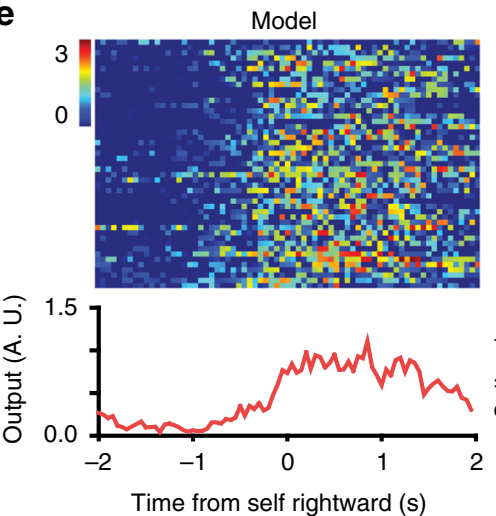

b

Distance

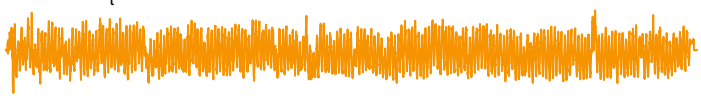

Right distance FSI (distance ${ }_{t-1}$ )

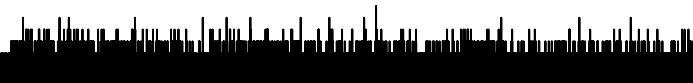

Difference (predicted velocity command from SPN)

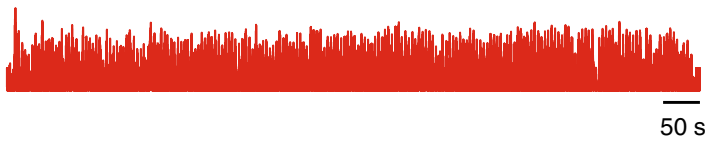

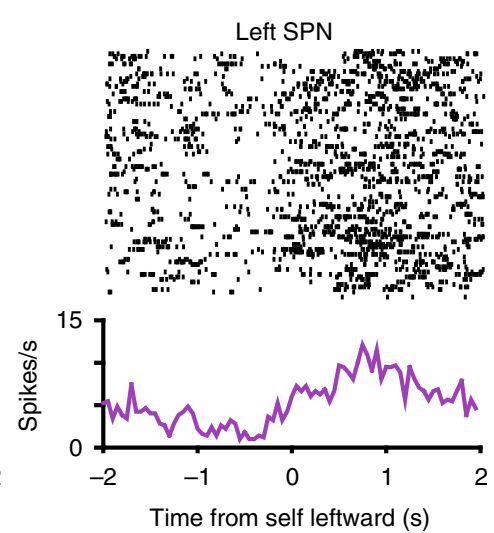

d

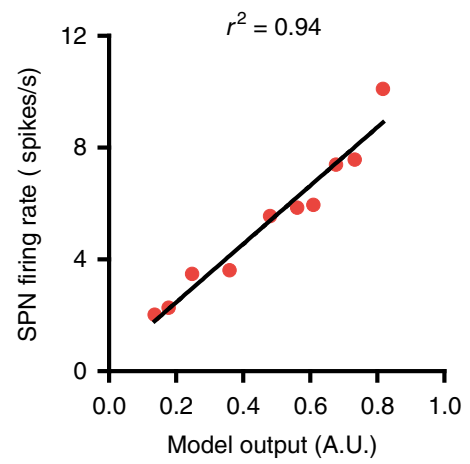

f

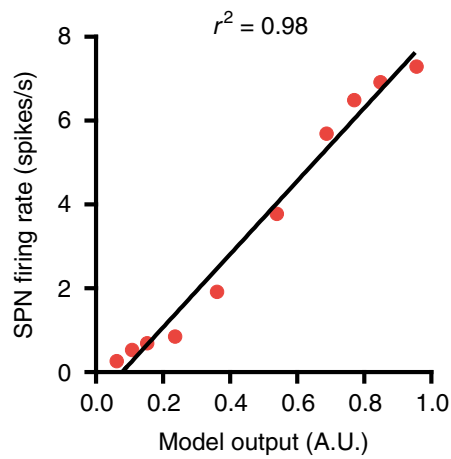

Fig. 7 Working model of how FSI-SPN circuit contributes to continuous pursuit. a Schematic diagram of the FSI-SPN feedforward inhibition circuit. Note that in the equation for velocity, distance refers to distance to target. This equation only applies to self-velocity during continuous pursuit. As shown here, excitatory cortical inputs reach both the FSI and SPN. In this case, some excitatory input represents distance to target, a key error signal used to guide pursuit behavior, and the FSI relays a slightly delayed version of the same signal to the SPN. Thus the delayed distance signal (Distance ${ }_{t-1}$ ) is subtracted from the distance signal (Distance ${ }_{t}$ ), and the SPN output reflects the difference between these two signals, i.e. the change in distance in this time step. As the feedforward inhibition circuit can function as a differentiator, the velocity command is proportional to the change in distance in a direction-specific manner. b Actual traces from our experiments illustrating the variables used in the model. c The model is compared with actual data using simultaneously recorded left distance FSI and left velocity SPN from the same mouse (same hemisphere). Using actual distance measure as an estimate of the Distance ${ }_{t}$ signal (shifted by $100 \mathrm{~ms}$ to account for the delay in the perceptual system) sent to the striatum, and FSI activity as the measure of the Distance $t_{-1}$ signal sent to the SPN, a subtraction generates a difference signal (model) that is compared to the activity of an actual left velocity SPN. d Model output is highly similar to right velocity SPN output (Pearson's $r, p<0.0001$. e Same as $\mathbf{c}$, except that the model is generated using a right distance FSI. The model is compared with actual data using simultaneously recorded right distance FSI and right velocity SPN. $\mathbf{f}$ High correlation between model output and right velocity SPN output $(p<0.0001)$. Source data are provided as a Source Data file

reflective markers $(6.35 \mathrm{~mm}$ diameter) were used for tracking mouse and target movements. One marker was located on the wireless recording headstage and another was $\sim 20 \mathrm{~mm}$ from the sucrose spout. These markers were captured by eight Raptor-H Digital Cameras (Motion analysis, CA, $100 \mathrm{~Hz}$ sampling rate). The data was transformed into Cartesian coordinates $(x, y$, and $z)$ by the Cortex program (Motion Analysis, CA). MATLAB communicated with the Cortex program
(Motion Analysis) online to control reward delivery based on mouse behavior. Reward ( $12 \mu \mathrm{l}$ of $20 \%$ sucrose) was delivered every $800 \mathrm{~ms}$ during following. Prior to the recording sessions, all mice were trained until they followed the target consistently (2-4 h of training over 1 week). In most experiments, the target moved at a constant velocity $(16 \mathrm{~mm} / \mathrm{s})$. On some sessions, however, target velocity was varied randomly $(5-48 \mathrm{~mm} / \mathrm{s}$, updated every $2 \mathrm{~ms}$ by MATLAB). 
Wireless in vivo electrophysiology. A total of $24 \mathrm{C} 57 \mathrm{BL} / 6 \mathrm{~J}$ mice were used in the electrophysiology experiments (17 males and 7 females). We used custom-built 16channel microwire arrays with micro-polished tungsten wires, $35 \mu \mathrm{m}$ in diameter and $4-5 \mathrm{~mm}$ in length, in a 2 by 8 configuration, and attached to an Omnetics connector (Omnetics Connector Corporation), as well as arrays with a similar configuration from Innovative Neurophysiology. To implant the electrode arrays, each mouse was anesthetized with isoflurane (induction at $3 \%$, maintained at $1 \%$ ), and head-fixed on a stereotax (Kopf). Meloxicam $(2 \mathrm{mg} / \mathrm{kg}$ ) was administered subcutaneously after anesthesia induction and prior to surgery for pain relief. Detailed surgery procedure was previously described ${ }^{43,58}$. The stereotaxic coordinates are (mm relative to Bregma): AP $+0.4 \mathrm{~mm}, \mathrm{ML} \pm 2.4 \mathrm{~mm}, \mathrm{DV}-2.3 \mathrm{~mm}$. Following the head implant surgeries, all mice were allowed to recover for at least 2 weeks before recording neural activity. Single unit activity was recorded with a miniaturized wireless headstage (Triangle Biosystems) and a Cerebus data acquisition system (Blackrock), as described previously ${ }^{59}$. The data was processed using online sorting algorithms and then re-sorted offline (Offline Sorter, Plexon). When classifying single unit waveforms, the following criteria were used: (1) a signal to noise ratio of at least $3: 1 ;(2)$ consistent waveforms throughout the recording session; and (3) refractory period of at least $800 \mu$ s. For correlation analysis, we first obtained rate histogram data in $10 \mathrm{~ms}$ bins. For FSIs, a $200 \mathrm{~ms}$ Gaussian filter was used for smoothing. For SPNs, a $50 \mathrm{~ms}$ Gaussian filter was used for smoothing. Correlation with each of the different behaviorally relevant variables (distance, velocity, and acceleration, and reward delivery) was computed. For reward correlation, neural and reward times were aligned by rightward self movement from $-3.5 \mathrm{~s}$ (left) to $3.5 \mathrm{~s}$ (right). Using different peri-event windows does not change the results. We compared the automatically computed correlation coefficients for different behavioral variables. A neuron is considered to be correlated with a particular behavioral variable if the absolute value of $r$ is higher than 0.75 and higher than the $r$ values for alternative variables.

In vivo calcium imaging. Five PV-Cre mice were used for calcium imaging experiments. Each mouse was unilaterally injected with $500 \mathrm{nl}$ of AAV-EF1a-DIOGCamp6s (Stanford virus core) into dorsolateral striatum (Bregma + 0.4, \pm 2.4 ; $-2.3 \mathrm{~mm}$ ) every 0.2 from $3.0 \mathrm{~mm}$ using a Nanoject III injector (Drummond Scientific, USA) at a rate of $1 \mathrm{nl} / \mathrm{s}$. The injection pipette was left in place after injection for $10 \mathrm{~min}$ before it was retracted. After viral injection, a gradient index (GRIN) lens (Inscopix: $1 \mathrm{~mm} \times 9 \mathrm{~mm}, n=3$; Grintech, $1.8 \mathrm{~mm} \times 4.2 \mathrm{~mm}, n=2$ ) was implanted in the dorsolateral striatum directly above the injection site after aspirating $\sim 2 \mathrm{~mm}$ of the above cortical tissue with 21-gauge blunt needle. The lens was then secured to the skull using dental cement and covered with Kwik-Sil to protect the lens surface. Two weeks after the GRIN lens implantation, the baseplate (Inscopix or UCLA Miniscope) was mounted onto the mouse head under visual guidance to determine the best field of view. All recorded videos of calcium activity were initially preprocessed in Mosaic (Inscopix) for motion correction and spatial binning ( $5 \times 5$ for Inscopix; $4 \times 4$ for miniscope), and subsequently analyzed using custom MATLAB scripts. We used constrained non-negative matrix factorization (CNMF) for denoising, deconvolving, and demixing of calcium-imaging data ${ }^{60,61}$. This method allows accurate source extraction of cellular signals.

TeLC experiments. Cre-inducible adeno-associated viral (AAV) plasmids containing GFP-tagged TeLC or GFP alone, each with the reading frame inverted in a flip-excision (FLEX) cassette (AAV-FLEX-TeLC and AAV-FLEX-GFP) were originally obtained from Dr. Peer Wulff, Christian Albrechts University, Kiel, Germany ${ }^{25}$, and the AAV vector was generated by the University of Pennsylvania vector core facility in serotype AAV2/5. We first implanted cannulae in PV-Cre mice $(n=13,24$ gauge, $3-\mathrm{mm}$ length below pedestal, Plastics One; AP $+0.4 \mathrm{~mm}$, $\mathrm{ML} \pm 2.4 \mathrm{~mm}, \mathrm{DV}-2.3 \mathrm{~mm}$ ). Cannulae were secured in place with skull screws and dental acrylic. A stylet was inserted and protruded $\sim 0.2 \mathrm{~mm}$ beyond the end of each cannula. The mice were then trained daily for $\sim 10$ days, each session consisting of 200 cycles of target movement. To inject the virus $(0.5 \mu \mathrm{l}$ of either TeLC-GFP or GFP virus), the stylets were removed, and virus was infused through custom made 31 gauge steel injectors extending $\sim 0.5$ beyond the tip of the guide cannulae at 0.1 $\mu \mathrm{l} / \mathrm{min}$ for $5 \mathrm{~min}$. The injector was kept in the brain for at least $5 \mathrm{~min}$ for diffusion. Coordinates for all injections relative to bregma were as follows: AP $+0.4 \mathrm{~mm}, \mathrm{ML}$ $\pm 2.4 \mathrm{~mm}, \mathrm{DV}-2.3 \mathrm{~mm}^{62}$. Approximately 3 weeks after surgery, the mice were tested on the pursuit task again.

DREADD experiments. The hM4Di virus $(n=6)$ or EYFP virus $(n=6)$ was injected into the sensorimotor striatum of Drdla-tdTomato::PV-Cre mice. The CAG-FLEX-rev-hM4D:2a:GFP plasmid was provided by Scott Sternson (Addgene \#52536). UNC Viral Vector Core packaged this plasmid into AAV $2 / 5$ and also provided AAV2/5-EF1a-DIO-EYFP (titers $>1 \times 10^{12}$ particles $/ \mathrm{mL}$ ). Small craniotomies were made over the injection sites and $1.0 \mu \mathrm{L}$ of virus was delivered bilaterally to dorsolateral striatum via a Nanoject II (Drummond Scientific) at a rate of $0.1 \mu \mathrm{L} / \mathrm{min}$. The injection pipette was held in place for $5 \mathrm{~min}$ following injection and then slowly removed. Coordinates for all injections relative to bregma were as follows: AP: $+0.8 \mathrm{~mm}, \mathrm{ML}: \pm 2.7 \mathrm{~mm}, \mathrm{DV}:-3.2 \mathrm{~mm}$. Mice were allowed a minimum of 3 weeks before behavioral training. The behavioral procedure is the same as described above, except mice were first tested on 100 cycles of target motion.
This pre-injection period establishes the baseline performance measures for each mouse, before given injection of the synthetic ligand $\mathrm{CNO}$ for the hM4Di receptor. CNO was dissolved to $10 \mathrm{mg} / \mathrm{mL}$ in DMSO and diluted in PBS solution to administer $5 \mathrm{mg} / \mathrm{kg}$ per mouse with a maximum injection volume of $0.5 \mathrm{~mL}$. The same volume of PBS/DMSO solution was used as the control vehicle injection. Thirty minutes after control or CNO injection, the mice were tested again for 100 cycles.

Optogenetics. To determine the effect of PV manipulation, $14 \mathrm{Pv}$-Cre mice were used in the optogenetic experiments (PV-Cre control, $n=4$; ChR2, $n=4$; stGtACR2, $n=6$ ). In addition, to determine the effect of SPN manipulation, 10 mice were used in optogenetic inhibition experiments (D1-cre::stGtACR2), $n=4$; A2A-Cre::stGtACR2), $n=3$; Control, $n=3$ ). AAV vectors were used for Credependent expression of the excitatory channelrhodopsin (pAAV-EFIa-DIOhChR2(H134R)-EYFP, Duke viral core, titer $>1 \times 10^{12}$ particles $/ \mathrm{mL}$ ), the inhibitory channelrhodopsin stGtACR2 (soma-targeted Guillardia theta anionconducting channelrhodopsin 2, pAAV_hSyn1-SIO-stGtACR2-FusionRed, titer > $1 \times 10^{13}$ particles $/ \mathrm{mL}$, Addgene). The vectors were injected ( $0.5 \mu \mathrm{l}$ each side) using the same procedure as described above using the following coordinates: $\mathrm{AP}+0.4$ $\mathrm{mm}, \mathrm{ML} \pm 2.4 \mathrm{~mm}, \mathrm{DV}-2.8 \mathrm{~mm}$. Both heterozygous male and female PV-Cre mice were used. Photo-stimulation is always bilateral. To measure in vivo neural activity during photo-stimulation, we implanted an optic fiber just above the site of injection (DV $-2.3 \mathrm{~mm}$ ) on one side and an optrode on the other side. The optrode was a custom made microwire array $(4 \mathrm{~mm}, 4 \times 4$ tungsten) with an optic fiber attached to the lateral side and angled so the cone of the emitted light encompassed the electrode tips ${ }^{63}$. Control mice received bilateral implants of optic fibers only. Before testing each day, mice were connected to a $2-\mathrm{m}$ sheathed fiber (105- $\mu \mathrm{m}$ core diameter, $0.22 \mathrm{NA}$ ) with a ceramic sleeve (Precision Fiber Products). The fiber was connected to a $470 \mathrm{~nm}$ DPSS laser (Shanghai Laser \& Optics), controlled with MATLAB (MathWorks). Before the experimental session, the final output of the laser was adjusted, based on the transmittance of each implant, to be $8-10 \mathrm{~mW}$ into the brain. Photo-stimulation $(500 \mathrm{~ms})$ was delivered $3.2 \mathrm{~s}$ after at least $800 \mathrm{~ms}$ of following. If the mouse continues to follow, stimulation was delivered every $3.2 \mathrm{~s}$. For optrode recordings, tethered setup was used (Cerebus data acquisition system, Blackrock).

Histology. After the completion of all behavioral tests, the mice with chronically implanted electrode arrays were deeply anesthetized with isoflurane and transcardially perfused with $0.9 \%$ saline followed by $10 \%$ buffered formalin solution. Coronal brain sections $(80 \mu \mathrm{m})$ were sliced using a Vibratome 1000 Plus, stained with thionin, and examined with a light microscope to verify location of the electrode tips within the striatum. All other mice were deeply anesthetized with isoflurane, perfused transcardially with Tris buffered saline (TBS; pH 7.4) containing $25 \mathrm{U} / \mathrm{ml}$ heparin, followed by $4 \%$ paraformaldehyde (PFA) in TBS. Perfused brains were removed, post-fixed overnight at $4{ }^{\circ} \mathrm{C}$ in $4 \%$ PFA, and then cryoprotected with $30 \%$ sucrose in TBS for $48 \mathrm{~h}$. Brains were cut into $50 \mu \mathrm{m}$ coronal sections using a cryostat (Leica CM 3000), and treated with blocking solution (TBS containing 5\% normal goat serum and $0.2 \%$ Triton $\mathrm{X}-100)$ for $2 \mathrm{~h}$ and incubated overnight at $4{ }^{\circ} \mathrm{C}$ with chicken anti-GFP monoclonal antibody (1:500; abcam; ab13970). After washing three times with TBST (TBS containing $0.2 \%$ Triton X$100)$, sections were incubated with goat anti-chicken Alexa Fluor ${ }^{\circledR} 488$ IgG (1:500 invitrogen; A11039) overnight at $4^{\circ} \mathrm{C}$. Sections were counterstained with a $4^{\prime}, 6-$ diamidino-2-phenylindole solution (DAPI; Sigma-Aldrich). After washing four times, the sections were cover slipped with FluorSave (CalBioChem) aqueous mounting medium. Whole brain section images were taken by tile scan imaging using a LSM 710 confocal microscope (Zeiss) with a $\times 10$ objective. To observe individual neurons expressing ChR2, stGtACR2, TelC, or DREADD, a $\times 20$ objective lens with $3 \times$ digital zoom was used.

Statistics. Statistical analysis was performed using Matlab and Graphpad Prism. No statistical methods were used to predetermine sample size. All statistical tests were two-tailed. To determine the effects of FSI inactivation, we used two-way repeated measures ANOVAs. Post hoc tests were performed whenever there was a significant interaction. To compute correlation between neural activity and behavioral variables, we used data from the entire session. First, we divided the data based on whether the animal was following the target, as described above. We then computed the task relevant behavioral variables, which include distance, velocity, and acceleration. We sorted the array of both firing rate and kinematics and excluded any outliers at the two extremes $(<1 \%$ or $>99 \%)$. We then divided the values into 20 bins and calculated correlation coefficient (Pearson's $r$ ) between the behavioral variables and neural activity using mean values from the bins. For the calcium imaging experiment, data was divided into six bins. For correlation with reward (sucrose drops/s), we calculated correlation coefficient only when animals were following because the reward was not delivered if they did not follow the target.

Reporting summary. Further information on research design is available in the Nature Research Reporting Summary linked to this article. 


\section{Data availability}

All data used in the present study are available upon request. Source data are provided as Source Data files.

\section{Code availability}

All Matlab codes used in the present study are available upon request.

Received: 3 December 2018 Accepted: 28 May 2019

Published online: 20 June 2019

\section{References}

1. Krauzlis, R. J. Recasting the smooth pursuit eye movement system. J. Neurophysiol. 91, 591-603 (2004).

2. Lisberger, S. G., Morris, E. \& Tychsen, L. Visual motion processing and sensory-motor integration for smooth pursuit eye movements. Annu. Rev. Neurosci. 10, 97-129 (1987).

3. O'Driscoll, G. A. et al. Functional neuroanatomy of smooth pursuit and predictive saccades. Neuroreport 11, 1335-1340 (2000).

4. Basso, M. A., Pokorny, J. J. \& Liu, P. Activity of substantia nigra pars reticulata neurons during smooth pursuit eye movements in monkeys. Eur. J. Neurosci. 22, 448-464 (2005)

5. Monteiro, P. \& Feng, G. Learning from animal models of obsessivecompulsive disorder. Biol. Psychiatry 79, 7-16 (2015).

6. Yin, H. H. \& Knowlton, B. J. The role of the basal ganglia in habit formation. Nat. Rev. Neurosci. 7, 464-476 (2006).

7. Gerfen, C. R. \& Wilson, C. J. The basal ganglia. In Handbook of chemical neuroanatomy (eds L. W. Swanson, A. Bjorklund \& T. Hokfelt) 371-468 (Elsevier, Amsterdam, 1996).

8. Mink, J. W. The basal ganglia: focused selection and inhibition of competing motor programs. Prog. Neurobiol. 50, 381-425 (1996).

9. Kravitz, A. V. et al. Regulation of parkinsonian motor behaviours by optogenetic control of basal ganglia circuitry. Nature 466, 622-626 (2010).

10. Gerfen, C. R., Baimbridge, K. G. \& Miller, J. J. The neostriatal mosaic: compartmental distribution of calcium-binding protein and parvalbumin in the basal ganglia of the rat and monkey. Proc. Natl. Acad. Sci. 82, 8780-8784 (1985).

11. Bolam, J. P., Hanley, J. J., Booth, P. A. \& Bevan, M. D. Synaptic organisation of the basal ganglia. J. Anat. 196, 527-542 (2000).

12. Tepper, J. M., Tecuapetla, F., Koos, T. \& Ibanez-Sandoval, O. Heterogeneity and diversity of striatal GABAergic interneurons. Front. Neuroanat. 4, 150 (2010).

13. Gage, G. J., Stoetzner, C. R., Wiltschko, A. B. \& Berke, J. D. Selective activation of striatal fast-spiking interneurons during choice execution. Neuron 67, 466-479 (2010).

14. Kalanithi, P. S. et al. Altered parvalbumin-positive neuron distribution in basal ganglia of individuals with Tourette syndrome. Proc. Natl. Acad. Sci. USA 102, 13307-13312 (2005)

15. Burguière, E., Monteiro, P., Feng, G. \& Graybiel, A. M. Optogenetic stimulation of lateral orbitofronto-striatal pathway suppresses compulsive behaviors. Science 340, 1243-1246 (2013).

16. O'Hare, J. K. et al. Striatal fast-spiking interneurons selectively modulate circuit output and are required for habitual behavior. eLife 6, e26231 (2017).

17. Kim, N., Barter, J. W., Sukharnikova, T. \& Yin, H. H. Striatal firing rate reflects head movement velocity. Eur. J. Neurosci. 40, 3481-3490 (2014).

18. Yttri, E. A. \& Dudman, J. T. Opponent and bidirectional control of movement velocity in the basal ganglia. Nature 533, 402-406 (2016).

19. Bartholomew, R. A. et al. Striatonigral control of movement velocity in mice. Eur. J. Neurosci. 43, 1097-1110 (2016).

20. Turner-Evans, D. et al. Angular velocity integration in a fly heading circuit. eLife 6, e23496 (2017).

21. Boyden, E. S., Zhang, F., Bamberg, E., Nagel, G. \& Deisseroth, K. Millisecondtimescale, genetically targeted optical control of neural activity. Nat. Neurosci. 8, 1263-1268 (2005).

22. Ghosh, K. K. et al. Miniaturized integration of a fluorescence microscope. Nat. Methods 8, 871-878 (2011)

23. Cai, D. J. et al. A shared neural ensemble links distinct contextual memories encoded close in time. Nature 534, 115-118 (2016).

24. Schiavo, G. et al. Tetanus and botulinum-B neurotoxins block neurotransmitter release by proteolytic cleavage of synaptobrevin. Nature 359, 832-835 (1992).

25. Murray, A. J. et al. Parvalbumin-positive CA1 interneurons are required for spatial working but not for reference memory. Nat. Neurosci. 14, 297-299 (2011).
26. Armbruster, B. N., Li, X., Pausch, M. H., Herlitze, S. \& Roth, B. L. Evolving the lock to fit the key to create a family of $\mathrm{G}$ protein-coupled receptors potently activated by an inert ligand. Proc. Natl Acad. Sci. USA 104, 5163-5168 (2007).

27. Zhang, F. et al. Multimodal fast optical interrogation of neural circuitry. Nature 446, 633-639 (2007)

28. Mahn, M. et al. High-efficiency optogenetic silencing with soma-targeted anion-conducting channelrhodopsins. Nat. Commun. 9, 4125 (2018).

29. Sohal, V. S., Zhang, F., Yizhar, O. \& Deisseroth, K. Parvalbumin neurons and gamma rhythms enhance cortical circuit performance. Nature 459, 698-702 (2009).

30. Zhang, F., Wang, L. P., Boyden, E. S. \& Deisseroth, K. Channelrhodopsin-2 and optical control of excitable cells. Nat. Methods 3, 785-792 (2006).

31. Ramanathan, S., Hanley, J. J., Deniau, J. M. \& Bolam, J. P. Synaptic convergence of motor and somatosensory cortical afferents onto GABAergic interneurons in the rat striatum. J. Neurosci. 22, 8158-8169 (2002).

32. Doig, N. M., Moss, J. \& Bolam, J. P. Cortical and thalamic innervation of direct and indirect pathway medium-sized spiny neurons in mouse striatum. J. Neurosci. 30, 14610-14618 (2010).

33. Wilson, C. J. Basal ganglia. In The Synaptic Organization of the Brain (ed. Shephard, G. M.) (Oxford University Press, New York, 2004).

34. Barter, J. W. et al. Basal ganglia outputs map instantaneous position coordinates during behavior. J. Neurosci. 35, 2703-2716 (2015).

35. Yin, H. H. The basal ganglia in action. Neuroscientist 23, 299-313 (2017)

36. Li, Y. et al. Hypothalamic Circuits for Predation and Evasion. Neuron 97, 911-924 e915 (2018).

37. Park, S. G. et al. Medial preoptic circuit induces hunting-like actions to target objects and prey. Nat. Neurosci. 21, 364-372 (2018).

38. Han, W. et al. Integrated control of predatory hunting by the central nucleus of the amygdala. Cell 168, 311-324 e318 (2017).

39. Kirouac, G. J., Li, S. \& Mabrouk, G. GABAergic projection from the ventral tegmental area and substantia nigra to the periaqueductal gray region and the dorsal raphe nucleus. J. Comp. Neurol. 469, 170-184 (2004).

40. Yin, H. H. Action, time and the basal ganglia. Philos. Trans. R. Soc. B 369, 20120473 (2014).

41. Barter, J. et al. Beyond reward prediction errors: the role of dopamine in movement kinematics. Front. Integr. Neurosci. 9, 39 (2015).

42. Barter, J. W., Castro, S., Sukharnikova, T., Rossi, M. A. \& Yin, H. H. The role of the substantia nigra in posture control. Eur. J. Neurosci. 39, 1465-1473 (2014).

43. Fan, D., Rossi, M. A. \& Yin, H. H. Mechanisms of action selection and timing in substantia nigra neurons. J. Neurosci. 32, 5534-5548 (2012)

44. Yin, H. H. How basal ganglia outputs generate behavior. Adv. Neurosci. 2014, 768313 (2014)

45. Yin, H. H. The role of opponent basal ganglia outputs in behavior. Future Neurol. 11, 149-169 (2016).

46. Bracci, E. \& Panzeri, S. Excitatory GABAergic effects in striatal projection neurons. J. Neurophysiol. 95, 1285-1290 (2006).

47. Isomura, Y. et al. Reward-modulated motor information in identified striatum neurons. J. Neurosci. 33, 10209-10220 (2013).

48. Owen, S. F., Berke, J. D. \& Kreitzer, A. C. Fast-spiking interneurons supply feedforward control of bursting, calcium, and plasticity for efficient learning. Cell 172, 683-695 e615 (2018).

49. Rueda-Orozco, P. E. \& Robbe, D. The striatum multiplexes contextual and kinematic information to constrain motor habits execution. Nat. Neurosci. 18, 453-460 (2015)

50. Klaus, A. et al. The Spatiotemporal organization of the striatum encodes action space. Neuron 95, 1171-1180 e1177 (2017).

51. Turner, R. S. \& Desmurget, M. Basal ganglia contributions to motor control: a vigorous tutor. Curr. Opin. Neurobiol. 20, 704-716 (2010).

52. Georgopoulos, A. P., DeLong, M. R. \& Crutcher, M. D. Relations between parameters of step-tracking movements and single cell discharge in the globus pallidus and subthalamic nucleus of the behaving monkey. J. Neurosci. 3, 1586-1598 (1983).

53. Gritton, H. J. et al. Unique contributions of parvalbumin and cholinergic interneurons in organizing striatal networks during movement. Nat. Neurosci. (2019).

54. Cui, G. et al. Concurrent activation of striatal direct and indirect pathways during action initiation. Nature 494, 238-242 (2013).

55. Rieke, F. Spikes: Exploring the Neural Code (The MIT Press, New York, 1999).

56. Geddes, C. E., Li, H. \& Jin, X. Optogenetic editing reveals the hierarchical organization of learned action sequences. Cell 174, 32-43 e15 (2018).

57. Ade, K. K., Wan, Y., Chen, M., Gloss, B. \& Calakos, N. An improved BAC transgenic fluorescent reporter line for sensitive and specific identification of striatonigral medium spiny neurons. Front. Syst. Neurosci. 5, 32 (2011).

58. Rossi, M. A., Fan, D., Barter, J. W. \& Yin, H. H. Bidirectional modulation of substantia nigra activity by motivational state. PLOS ONE 8, e71598 (2013).

59. Fan, D. et al. A wireless multi-channel recording system for freely behaving mice and rats. PLoS ONE 6, e22033 (2011). 
60. Zhou, P. et al. Efficient and accurate extraction of in vivo calcium signals from microendoscopic video data. eLife 7, e28728 (2018).

61. Pnevmatikakis, E. A. et al. Simultaneous denoising, deconvolution, and demixing of calcium imaging data. Neuron 89, 285-299 (2016).

62. Paxinos, G. \& Franklin, K. The Mouse Brain in Stereotaxic Coordinates. (Academic Press, New York, 2003).

63. Sparta, D. R. et al. Construction of implantable optical fibers for long-term optogenetic manipulation of neural circuits. Nat. Protoc. 7, 12-23 (2012).

\section{Acknowledgements}

We would like to thank Justin O'Hare and Nicole Calakos for their help with DREADD experiments, Koji Toda and Dongye Lu for help with 3D printing, Scott Soderling for providing the Inscopix microscope for some of the calcium-imaging experiments, and Mark Rossi and Konstantin Bakhurin for helpful comments on the manuscript. This research was supported by grants from the National Institutes of Health (DA040701, NS094754, MH112883 to H.H.Y., DA033610 to A.E.W., and MH117429 to I.H.K.).

\section{Author contributions}

N.K. and H.H.Y. designed the experiments. D.G. and A.E.W. provided reagents. N.K. H.E.L., R.N.H., G.D.R.W. and I.H.K. conducted experiments. N.K. and H.H.Y. analyzed the data and wrote the manuscript.

\section{Additional information}

Supplementary Information accompanies this paper at https://doi.org/10.1038/s41467019-10716-w.

Competing interests: The authors declare no competing interests.
Ethical compliance: All research reported in this study was approved by Duke University Institutional Animal Care and Use Committee (protocol A263-16-12).

Reprints and permission information is available online at http://npg.nature.com/ reprintsandpermissions/

Peer review information: Nature Communications thanks Ivan de Araujo, Charles Gerfen and other anonymous reviewer(s) for their contribution to the peer review of this work.

Publisher's note: Springer Nature remains neutral with regard to jurisdictional claims in published maps and institutional affiliations.

(c) (i) Open Access This article is licensed under a Creative Commons Attribution 4.0 International License, which permits use, sharing, adaptation, distribution and reproduction in any medium or format, as long as you give appropriate credit to the original author(s) and the source, provide a link to the Creative Commons license, and indicate if changes were made. The images or other third party material in this article are included in the article's Creative Commons license, unless indicated otherwise in a credit line to the material. If material is not included in the article's Creative Commons license and your intended use is not permitted by statutory regulation or exceeds the permitted use, you will need to obtain permission directly from the copyright holder. To view a copy of this license, visit http://creativecommons.org/ licenses/by/4.0/.

(C) The Author(s) 2019 